\title{
1 Relationship between simultaneously recorded spiking activity and fluorescence signal in GCaMP6 transgenic mice
}

3

4

5 Authors

6 Lawrence Huang $^{1 *}$, Peter Ledochowitsch ${ }^{1 *}$, Ulf Knoblich ${ }^{1}$, Jérôme Lecoq ${ }^{1}$, Gabe J. Murphy ${ }^{1}$, R.

7 Clay Reid ${ }^{1}$, Saskia E. J. de Vries ${ }^{1}$, Christof Koch ${ }^{1}$, Hongkui Zeng ${ }^{1 \#}$, Michael A. Buice ${ }^{1}$, Jack

8 Waters $^{1 \#}, \mathrm{Lu} \mathrm{Li}^{1,2,3,4 \#}$

9

101 Allen Institute for Brain Science, Seattle, WA 98109, USA

112 Guangdong Provincial Key Laboratory of Malignant Tumor Epigenetics and Gene

12 Regulation, Guangdong-Hong Kong Joint Laboratory for RNA Medicine, Sun Yat-sen

13 Memorial Hospital, Sun Yat-sen University, Guangzhou, Guangdong Prov., China 510120

143 Medical Research Center, Sun Yat-sen Memorial Hospital, Sun Yat-sen University,

15 Guangzhou, Guangdong Prov., China 510120

164 Centre for Brain Science and Brain-Inspired Intelligence, Guangdong-Hongkong-Macao

17 Greater Bay Area, China

* These authors contributed equally.

21 jackw@alleninstitute.org (J.W.) or lilu67@mail.sysu.edu.cn (L.L.).

\section{Keywords}

24 calcium imaging, genetically encoded calcium indicator, action potential, excitatory neurons, 25 calibration 


\section{ABSTRACT}

Fluorescent calcium indicators are often used to investigate neural dynamics, but the relationship between fluorescence and action potentials (APs) remains unclear. Most APs can be detected when the soma almost fills the microscope's field of view, but calcium indicators are often used to image populations of neurons, necessitating a large field of view, generating fewer photons per neuron, and compromising AP detection. Here we characterized the AP-

fluorescence transfer function in vivo for 48 layer 2/3 pyramidal neurons in primary visual cortex, with simultaneous calcium imaging and cell-attached recordings from transgenic mice expressing GCaMP6s or GCaMP6f. While most APs were detected under optimal conditions, under conditions typical of population imaging studies only a minority of 1AP and 2AP events were detected (often $<10 \%$ and $\sim 20-30 \%$, respectively), emphasizing the limits of AP detection under more realistic imaging conditions.

\section{INTRODUCTION}

Genetically encoded calcium indicators (GECls) are widely used with 2-photon laser scanning microscopy to report neuronal activity within local populations in vivo (Luo et al., 2018). This optical approach is minimally invasive and enables simultaneous measurement of activity from hundreds or even thousands of neurons at single-cell resolution, over multiple sessions. Using a contemporary GECl such as GCaMP6s, fluorescence changes associated with isolated spikes (action potentials, APs) in vivo can be detected when imaged at sufficiently high spatiotemporal resolution (Chen et al., 2013) (http://dx.doi.org/10.6080/K02R3PMN). Yet undetected APs are common in population imaging experiments (Theis et al., 2016; Berens et al., 2018).

Inferring the underlying AP train or firing rate from calcium imaging remains challenging for several reasons. Firstly, population imaging studies necessarily employ a large field of view containing many neurons. In contrast, the AP to calcium-dependent fluorescence transfer function is typically characterized with a soma filling the field of view of the microscope, to maximize photon flux from the soma and thereby signal-to-noise ratio. Secondly, there is no ground truth spiking information available for most neurons in a population. Spiking information, often from a cell-attached recording, can be used to refine the spike inference model and thereby optimize AP detection. Thirdly, the AP to calcium-dependent fluorescence transfer

58 function may be different for each neuron due to various intrinsic and extrinsic factors, such as neuron-to-neuron differences in indicator expression. 
61 Compared to viral expression, transgenic mouse lines offer convenience (e.g. bypassing virus

62 injection and associated procedures) and achieve more uniform $\mathrm{GECl}$ expression in genetically

63 defined neuronal populations (Madisen et al., 2015; Daigle et al., 2018). Using our intersectional

64 transgenic mouse lines that enable Cre recombinase-dependent expression of GCaMP6s or

65 GCaMP6f, we simultaneously characterized the spiking activity and fluorescence of individual

66 GECl-expressing pyramidal neurons in layer 2/3 of mouse primary visual cortex (V1). We then

67 tested the performance of several spike inference models, detecting APs under optimal

68 conditions (models refined using the spiking information, with the soma filling the field of view)

69 and under the less optimal conditions typical of population imaging experiments. Our results

70 provide insight into the relationship between spiking activity in vivo and fluorescence signals and

71 will aid the interpretation of existing and future calcium imaging datasets.

RESULTS

75

To characterize the single-cell transfer function between observed fluorescence signals and underlying APs in vivo, we performed simultaneous calcium imaging and cell-attached recordings in V1 L2/3 excitatory pyramidal neurons in anesthetized mice (Figure 1A,B). To directly compare our results to virally-expressed GCaMP6f and GCaMP6s (Chen et al., 2013) (http://dx.doi.org/10.6080/K02R3PMN), we used a small field of view (19.3-27.3 x 19.3-21.5 $\mu$; scanning rate 141.3-158.3 frames per second, fps). 2-10 minute recordings were obtained from 213 neurons, all with fluorescence excluded from the nucleus. Quality control code was deployed to exclude from further analysis recordings with artifacts such as motion, photobleaching, somatic dye from the recording pipette, electrophysiological or fluorescence baseline instability, and abrupt changes in AP waveform (see Methods). The dataset for further analysis was from 48 neurons from mice of 4 transgenic lines, two expressing GCaMP6s and two GCaMP6f in excitatory neurons in layer 2/3 and deeper layers of cortex (Table 1).

We analyzed events with fluorescence transients separated from those of adjacent events, containing a total of 5427 APs (28\% of APs; Figure 1 - figure supplement $1 \mathrm{C}$ ). An event was defined as one or more APs within $250 \mathrm{~ms}$, with no APs in the preceding or subsequent $300 \mathrm{~ms}$

91 for GCaMP6f or $1 \mathrm{~s}$ and $500 \mathrm{~ms}$ for GCaMP6s (Figure 1C).

\section{Calcium transients differ across mouse lines}


Fluorescence measured from the soma is contaminated with fluorescence from the surrounding neuropil, due to the extended nature of the microscope point spread function. Neuropil contamination is often removed by subtracting a scaled version of the neuropil fluorescence from the somatic fluorescence, with the scale factor referred to as the $r$ value (Kerlin et al., 2010; Akerboom et al., 2012). The r value can affect AP detection, with under-subtraction of neuropil leading to false positives (events detected when there was activity in the neuropil but not the soma) and over-subtraction to false negatives (failure to detect somatic activity). We examined the effects of $r$ on detection of $1 \mathrm{AP}$ events, with electrical recordings providing ground truth (Figure 2 and Figure 2 - figure supplement 1). For many GCaMP6s neurons, the receiver operating characteristic ( $R O C$ ) curve changed little with $r$ (Figure $2 A)$, indicating that APs were detected with few false positives with little effect of neuropil subtraction. Neuropil subtraction exerted a stronger influence on event detection in GCaMP6f neurons, where the ROC curve changed with $r$ (Figure 2A), permitting identification of the optimal $r$ as that which maximized the area under the ROC curve and, thereby, the true/false event detection ratio. Optimal $r$ for Emx1$f$ and Cux2-f neurons was approximately normally distributed with mean \pm sem of $0.82 \pm 0.07$ (20 neurons, Figure 2B). Our results indicate that the value of $r$ has a modest effect on event detection in GCaMP6f neurons in mouse V1. The effect of neuropil subtraction may be greater 112 during coordinated activity across the whole network, such as during strong sensory stimuli.

After neuropil subtraction (see Methods), we averaged trials by number of APs, fit a sum of exponentials to estimate rise and decay time constants and calculated peak $\Delta F / F$ (mean fluorescence over $100 \mathrm{~ms}$ around the maximum within $300 \mathrm{~ms}$ for GCaMP6f and $500 \mathrm{~ms}$ for GCaMP6s) for events with 1-5 APs (Figure 3). 28-55\% of detected APs were in events with 1-5 APs (Figure 1 - figure supplement 1 ) and $>70 \%$ of these analyzed APs were in multi-AP events. As expected, peak $\Delta F / F$ increased approximately linearly with 1-5 APs, and peak $\Delta F / F$ and decay time constant were greater with GCaMP6s than GCaMP6f (Figure 3C). Peak $\Delta F / F$ was comparable to or slightly greater than in previous studies with GCaMP6s and GCaMP6f,

122 ( $r=0.8$ vs 0.7; Chen et al., 2013).

123

As expected, photon shot noise was the dominant noise source in images from all mouse lines. The pixelwise slope of the least squares fit between the variance and mean of the photon flux was $1.04 \pm 0.01$ (mean \pm sem), consistent with the noise following a Poisson process (intercept $-0.08 \pm 0.2,48$ neurons). Trial-to-trial variability in the amplitude of the 1 AP-evoked fluorescence 
was substantial and exceeded photon shot noise in most neurons (Figure 3 - figure supplement 1). The sources of non-Poisson variability in our results are unclear, but negligible motion was visible in the movies after motion correction. Likely the variability results primarily from trial-totrial differences in the AP-evoked calcium concentration and GCaMP6f and GCaMP6s are expressed at sufficient concentrations to report resting changes in calcium concentration in all four mouse lines.

\section{Increasing field of view reduces optimized event detection} GCaMP6 indicators have been widely adopted because they exhibit greater AP-evoked $\Delta F / F$ than previous GCaMP indicators, but still some APs may go undetected (Chen et al., 2013). Under ideal conditions, almost all APs can be detected (with probability close to 1 at a false positive probability of $1 \%$; Chen et al., 2013). However, many imaging experiments are performed with a field of view of hundreds of micrometers and this large field of view limits the dwell time per soma and thereby the photon flux per soma and signal-to-noise ratio. What event detection rate might be expected when imaging a large field of view, sufficient to include hundreds of somata? How much does field of view affect event detection?

\section{We calculated detection probability for $1 \mathrm{AP}$ and $2 \mathrm{AP}$ events, using AP times from} electrophysiology recordings to optimize event detection for each neuron (Chen et al., 2013). In high spatial and temporal resolution images, the probability of 1AP event detected spanned a wide range (probability 0.07-1 and 0.11-0.95 for GCaMP6s and GCaMP6f, at 1\% false positive probability, Figure 5A-C). As expected (Dana et al., 2014; Wei et al., 2019), most 1AP events were detected in GCaMP6s and GCaMP6f neurons, but with lower average probability in GCaMP6f neurons (1AP detection probability $0.70 \pm 0.06$ for 18 Emx1-s neurons, $0.80 \pm 0.03$ for 3 tetO-s neurons, $0.40 \pm 0.08$ for 9 Cux2-f neurons, $0.60 \pm 0.08$ for 11 Emx 1 -f neurons, mean \pm sem at $1 \%$ false positive probability). 2AP events were reliably detected in all four mouse lines (Figure 5C; detection probability $0.90 \pm 0.06$ for Emx1-s, $1.0 \pm 0.0$ for tetO-s, $0.66 \pm$ 0.07 for Cux2-f, $0.80 \pm 0.05$ for Emx1-f, at $1 \%$ false positive probability). In high spatial and temporal resolution images, in all four mouse lines it was possible to detect most but by no means all 1 AP and 2 AP events with a false positive probability of only $1 \%$.

In these transgenic mice, 1AP detection probabilities were lower than in previously reported neurons with virally-expressed GCaMP6s and GCaMP6f $(0.99$ and 0.84 at $1 \%$ false positive probability in Chen et al., 2013). There are several possible reasons for this difference. In the 
transgenic mice used here, GCaMP expression is widespread throughout neocortex, which may result in labeling of greater numbers of axons and dendrites that contribute to the neuropil signal. Furthermore, GCaMP6 expression may be weaker in the four TIGRE1.0 mouse line crosses examined here than with strong promoter-driven adeno-associated virus (AAV) vectors as used in Chen et al. (2013). The newer TIGRE2.0 reporter lines drive GCaMP expression that's comparable to that from strong promoter-driven AAVs (Daigle et al., 2018), likely enabling $1 \mathrm{AP}$ and $2 \mathrm{AP}$ detection rates in transgenic mice that are comparable to those achieved with viral expression of GCaMP6.

Our recordings were obtained with a small field of view, at a high frame rate and centered on the soma $(\sim 19.3 \times 19.3 \mu \mathrm{m}, \sim 158 \mathrm{~Hz}$, Figure 4A,B). In an attempt to simulate commonly used imaging conditions, we downsampled our images in space and time to mimic imaging with a 412 x $412 \mu \mathrm{m}$ field of view at $30.3 \mathrm{~Hz}$, as used in the Allen Brain Observatory (Figure 4C,D). The baseline fluorescence noise from downsampled images was comparable to that in the Allen Brain Observatory (Figure 4E) and is presumably comparable to images in many 2-photon datasets with populations of hundreds of neurons.

As expected, event detection probabilities were lower for downsampled images than for the original, high resolution images (Figure 5D-F). 1AP and 2AP event detection probabilities were $0.32 \pm 0.05$ and $0.55 \pm 0.08$ for $18 \mathrm{Emx1}$-s neurons, $0.43 \pm 0.07$ and $0.89 \pm 0.06$ for 3 tetO-s neurons, $0.16 \pm 0.04$ and $0.24 \pm 0.04$ for 9 Cux2-f neurons, $0.21 \pm 0.04$ and $0.42 \pm 0.09$ for 11 Emx1-f neurons (mean \pm sem at $1 \%$ false positive probability). Even for 2AP events, detection probability is $<0.5$ when imaging with GCaMP6f and a field of view of several hundred micrometers.

In summary, 1AP and 2AP events were detected with high probability when images were acquired with high spatial and temporal resolution and when analysis was performed with an event detection algorithm optimized for each neuron using known AP times. Even with known AP times to optimize detection for each neuron, event detection was severely impaired by a reduction in spatial and temporal resolution to mimic a typical 2-photon population imaging experiment.

\section{Modest effect of field of view on event detection under typical imaging conditions}


In a typical 2-photon population imaging experiment, no electrophysiology recording is available to optimize event detection. Often, the shape of the calcium transient is estimated from published indicator rise and decay times or derived from a representative sample of fluorescence transients. What are typical event detection and false positive probabilities under these sub-optimal conditions, blind to the underlying AP times? Is performance degraded equally for high and for low resolution images?

We compared event detection from high resolution and downsampled images using three spike inference algorithms: unconstrained non-negative deconvolution (NND; Friedrich et al., 2017), non-negative convolution with an LO constraint to enforce event sparseness (Exact L0; Jewell et al., 2020) and a biophysical model that explicitly accounts for intracellular calcium dynamics (MLspike; Deneux et al., 2016). These three algorithms are among the highest performing spike inference algorithms (Berens et al., 2018).

For each neuron, the algorithms were deployed to estimate the number of APs in each image of the movie. All three algorithms estimated AP numbers that approximately recapitulated the number of APs measured with electrophysiology, but the number of APs per frame was typically not an integer due to imperfect spike inference (Figure 6A,D). We characterized performance using the Pearson correlation coefficient and the Matthews correlation coefficient, which compare measured and estimated AP number at each time point, and the presence or absence of an event at each time point, respectively. Pearson correlation coefficients were $\sim 0.4$ when calculated with $33 \mathrm{~ms}$ time bins, increasing towards 0.7 as bin size was increased to $500 \mathrm{~ms}$ (Figure 6 - figure supplement 1), comparable to published results (Berens et al., 2018). Mean Pearson and Matthews correlation coefficients were similar across inference algorithms and mouse lines and differed little between high resolution and downsampled images (Figure 6B,E).

We plot ROC curves to more directly examine the relationship between detected events and false positives. Since spike inference is generally useful only where false positive rates are low, we focused on false positive probabilities in the range of 0-0.05. Performance differed greatly between neurons, but mean ROC curves were similar across mouse lines, with only modest differences between algorithms, between GCaMP6s and GCaMP6f lines or between high resolution and downsampled conditions (Figure 6C,F). 
228 Naturally, detection probability increased with the number of APs per event. At a false positive

229 probability of 0.01 , detection probability was commonly $<0.1$ for $1 \mathrm{AP}$ events, increasing

230 approximately linearly with AP number, often to $\sim 0.8$ for 5AP events (Figure 7A,B). With 1AP

231 events being the most common event type in all four mouse lines (Figure 7C), it was possible to

232 detect only a minority of events with a low false positive probability. Using these spike inference

233 algorithms, although detection probabilities were commonly slightly lower for downsampled than

234 for high resolution images, the difference was modest, indicating that the decreased SNR of

235 population imaging had little effect on event detection in our dataset.

Using our dataset, we compared event detection with three algorithms: non-negative deconvolution (NND), non-negative deconvolution with an exact LO constraint (Exact LO) and the biophysically inspired MLspike model. For NND, performance was poor at $30 \mathrm{~Hz}$ and considerably improved by upsampling to $150 \mathrm{~Hz}$ (Figure 7 - figure supplement 1). Upsampling of low frame rate data, often to $100 \mathrm{~Hz}$, is a common practice in the field (Theis et al., 2016; Berens et al., 2018; Pachitariu et al., 2018). For MLspike, performance was poor without use of the autocalibration procedure to optimize the model for each neuron (Figure 7 - figure supplement 2). MLspike thus contrasted with deconvolution-based algorithms, for which fixed parameters are more effective (Pachitariu et al., 2018). For Exact L0, neither upsampling nor optimization for each neuron was necessary for optimal mean performance across neurons.

Pachitariu et al. (2018) found that non-negative deconvolution (NND) matched and often exceeded the performance of more constrained algorithms such as NND with an approximate LO constraint. Consistent with the conclusions of Pachitariu et al. (2018), Exact LO often lagged the performance of NND (Figure 7). The performance of MLspike was broadly equivalent to that of NND, but the loss of performance due to downsampling was less with MLspike, resulting in outperformance of MLspike on downsampled images. Our results point to MLspike as a compelling choice for spike inference in population imaging experiments. Our results also suggest there's ample room for improvement of spike inference models since event detection by the three spike inference models falls far short of the performance of the ground truth optimized approach employed in figure 5 (Figure 7 - figure supplement 3 ).

In summary, relative to small field of view imaging, population imaging conditions decreased the probability of spike event detection with an event detector optimized to each individual neuron using ground truth AP information (Figure 5). With blind spike inference, many events went 
undetected even under near-ideal imaging conditions with a small field of view, and event detection was not substantially worse under population imaging conditions (Figure 6, Figure 7, Figure 7 - figure supplement 3). The results of Figures 6 and 7 are likely representative of event detection in many GCaMP6 imaging experiments, where ground truth AP information is not available and blind spike inference is employed. Our results indicate that even though GCaMP6 indicators are bright and sensitive enough to enable the detection of most $1 \mathrm{AP}$ events in superficial cortical pyramidal neurons in vivo if the detection procedure is optimized using ground truth AP information, most events containing 1,2 and sometimes greater numbers of APs go undetected in our and likely many other imaging experiments with GCaMP6.

\section{DISCUSSION}

Calcium imaging is widely used to report neuronal spiking activity in vivo. However, accurate spike inference from calcium imaging remains a challenge, and there are relatively few ground truth datasets with simultaneous calcium imaging and electrophysiology to aid the development of more accurate spike inference algorithms. In a recent challenge (Spike Finder;

http://spikefinder.codeneuro.org/) (Berens et al., 2018), 40 algorithms were trained and tested on datasets consisting of 37 GCaMP6-expressing neurons, underscoring the need for additional GCaMP6 calibration data (Berens et al., 2018). In addition to supporting efforts toward spike inference, an improved understanding of the relationship between spiking and observed fluorescence signals is necessary to further broaden the utility and impact of calcium imaging. To these ends, we contribute a ground truth dataset consisting of 48 V1 L2/3 excitatory neurons recorded at single-cell resolution (available at https://portal.brainmap.org/explore/circuits/oephys), and characterized their AP-to-calcium fluorescence transfer function. Complementing existing datasets with viral GECI expression (Chen et al., 2013; Theis et al., 2016; Dana et al., 2016), our work facilitates interpretation of existing and future calcium imaging studies using mainstream transgenic mouse lines, such as the Allen Institute's Brain Observatory Visual Coding dataset (http://observatory.brain-map.org/visualcoding) (de Vries et al., 2020).

Previous studies have established that most APs can be detected with GCaMP6 indicators under near-optimal conditions (Chen et al., 2013). Yet undetected APs are common in population imaging experiments (Theis et al., 2016; Berens et al., 2018). To investigate why APs are often missed during population imaging, we compared event detection in 250 ms time- 
windows with a neuronal soma occupying most of the image, near-optimal conditions for AP event detection, and event detection when the soma occupies just a small percentage of the field of view, less ideal conditions that are common in population imaging studies. Importantly, we downsampled images to simulate population imaging conditions, enabling comparison for the same APs under different imaging conditions.

Our results indicate that, in GCaMP6 transgenic mice, most APs can be detected under nearoptimal conditions, while detection is less effective during population imaging. These conclusions are similar to those of previous studies with viral GCaMP6 expression, but our results also reveal two reasons for the difference in detection. Unsurprisingly, the reduced signal-to-noise ratio of population imaging, relative to single soma imaging, results in less effective event detection. However, a high signal-to-noise ratio, achieved by imaging one soma, was no guarantee of effective event detection. Effective detection also required optimization of detection for the neuron of interest, using known AP times to identify events with different AP numbers and so generate kernels of the appropriate amplitude and time course. Parameter tuning in the absence of known AP times, with the MLspike autocalibration routine, improved event detection but not to the high standard of ground truth-optimized detection. Unfortunately, measuring AP times for every neuron with electrophysiology is rarely feasible, severely limiting the percentage of events one might reasonably expect to detect with GCaMP6 in most imaging experiments.

Our results point to several practices that might be adopted to maximize spike detection. Firstly, minimize the field of view, maximizing photon flux per neuron. Secondly, tune the spike inference model for each neuron independently, where possible. Thirdly, compare the results of several spike inference models. The 3 models employed here produced similar AP detection rates, whether applied to high resolution or to downsampled images. Similarly, Pachitariu et al. (2018) observed that the L0 constraint failed to improve performance of the NND model. Nonetheless, each model has strengths and weaknesses. For example, a model may detect more APs than another but at the cost of a greater false positive rate. As a result, model performance may diverge for some AP rates and patterns. In the worst case, comparing models provides some protection from errors in implementation. Fourthly, ensure that traces are sampled (or upsampled) at a sufficiently high rate when employing NND and use autocalibration with MLspike; both make a substantial difference to model performance. Finally, exercise 
caution when interpreting the inferred spike rates. Commonly, many APs are not detected using even the most accurate spike inference models.

332 In summary, in this study we present a ground truth dataset from anesthetized mice with

333 simultaneous electrophysiology and calcium imaging. Analysis of this dataset revealed that only

334 a small fraction of isolated action potentials were detected under typical population imaging

335 conditions and with existing spike inference algorithms. By making our data freely available, we

336 hope that it will serve the community as a further resource to better understand the quantitative

337 link between calcium-evoked fluorescent imaging signals and spiking activity.

MATERIALS AND METHODS

Key Resources Table

\begin{tabular}{|c|c|c|c|c|}
\hline $\begin{array}{l}\text { Reagent } \\
\text { type } \\
\text { (species) or } \\
\text { resource }\end{array}$ & Designation & $\begin{array}{l}\text { Source or } \\
\text { reference }\end{array}$ & Identifiers & $\begin{array}{l}\text { Additional } \\
\text { information }\end{array}$ \\
\hline $\begin{array}{l}\text { Genetic } \\
\text { reagent } \\
\text { (Mus } \\
\text { musculus) }\end{array}$ & $\begin{array}{l}\text { B6.129S2-Emx1tm1(cre)Krj/J, } \\
\text { Emx1-IRES-Cre }\end{array}$ & $\begin{array}{l}\text { Jackson } \\
\text { Laboratory }\end{array}$ & $\begin{array}{l}\text { RRID:IMSR_JA } \\
\text { X:005628 } \\
\text { RRID:MGI:2684 } \\
610\end{array}$ & \\
\hline $\begin{array}{l}\text { Genetic } \\
\text { reagent } \\
\text { (Mus } \\
\text { musculus) }\end{array}$ & $\begin{array}{l}\text { B6(Cg)- } \\
\text { Cux2tm3.1(cre/ERT2)Mull/Mm } \\
\text { mh, } \\
\text { Cux2-CreERT2 }\end{array}$ & MMRRC & $\begin{array}{l}\text { RRID:MMRRC_ } \\
\text { 032779-MU } \\
\text { RRID:MGI:5014 } \\
172\end{array}$ & \\
\hline $\begin{array}{l}\text { Genetic } \\
\text { reagent } \\
\text { (Mus } \\
\text { musculus) }\end{array}$ & $\begin{array}{l}\text { B6.Cg-Tg(Camk2a- } \\
\text { tTA)1Mmay/DboJ, } \\
\text { Camk2a-tTA }\end{array}$ & $\begin{array}{l}\text { Jackson } \\
\text { Laboratory }\end{array}$ & $\begin{array}{l}\text { RRID:IMSR_JA } \\
\text { X:007004 } \\
\text { RRID:MGI:2179 } \\
066\end{array}$ & \\
\hline $\begin{array}{l}\text { Genetic } \\
\text { reagent } \\
\text { (Mus } \\
\text { musculus) }\end{array}$ & $\begin{array}{l}\text { B6;DBA-Tg(tetO- } \\
\text { GCaMP6s)2Niell/J, } \\
\text { tetO-GCaMP6s }\end{array}$ & $\begin{array}{l}\text { Jackson } \\
\text { Laboratory }\end{array}$ & $\begin{array}{l}\text { RRID:IMSR_JA } \\
\text { X:024742 } \\
\text { RRID:MGI:5553 } \\
332\end{array}$ & \\
\hline $\begin{array}{l}\text { Genetic } \\
\text { reagent } \\
\text { (Mus } \\
\text { musculus) }\end{array}$ & $\begin{array}{l}\text { B6;129S6-Igs7tm93.1(tetO- } \\
\text { GCaMP6f)Hze/J, } \\
\text { Ai93(TITL-GCaMP6f) }\end{array}$ & $\begin{array}{l}\text { Jackson } \\
\text { Laboratory }\end{array}$ & $\begin{array}{l}\text { RRID:IMSR_JA } \\
\text { X:024103 } \\
\text { RRID:MGI:5558 } \\
086\end{array}$ & \\
\hline
\end{tabular}




\begin{tabular}{|l|l|l|l|l|}
\hline $\begin{array}{l}\text { Genetic } \\
\text { reagent } \\
\begin{array}{l}\text { (Mus } \\
\text { musculus) }\end{array}\end{array}$ & $\begin{array}{l}\text { B6.Cg-Igs7tm94.1(tetO- } \\
\text { GCaMP6s)Hze/J, } \\
\text { Ai94(TITL-GCaMP6s) }\end{array}$ & $\begin{array}{l}\text { Jackson } \\
\text { Laboratory }\end{array}$ & $\begin{array}{l}\text { RRID:IMSR_JA } \\
\text { X:024104 } \\
\text { RRID:MGI:5607 } \\
576\end{array}$ & \\
\hline $\begin{array}{l}\text { Software, } \\
\text { algorithm }\end{array}$ & MATLAB R2016b & $\begin{array}{l}\text { http://www. } \\
\text { mathworks } \\
\text { com/produ } \\
\text { cts/matlab/ }\end{array}$ & $\begin{array}{l}\text { RRID:SCR_001 } \\
622\end{array}$ & \\
\hline $\begin{array}{l}\text { Software, } \\
\text { algorithm }\end{array}$ & Python 3.7.4 & $\begin{array}{l}\text { http://www. } \\
\text { python.org/ }\end{array}$ & $\begin{array}{l}\text { RRID:SCR_008 } \\
394\end{array}$ & \\
\hline $\begin{array}{l}\text { Software, } \\
\text { algorithm }\end{array}$ & LabVIEW 2015 & $\begin{array}{l}\text { http://www. } \\
\text { ni.com/labv } \\
\text { iew/ }\end{array}$ & $\begin{array}{l}\text { RRID:SCR_014 } \\
\text { 325 }\end{array}$ & \\
\hline
\end{tabular}

342

343

344

345

346

347

348

349

350

351

352

353

354

355

356

357

358

359

360

361

362

363

Experimental procedures were conducted in accordance with NIH guidelines and approved by the Institutional Animal Care and Use Committee (IACUC) of the Allen Institute for Brain Science under protocol number 1509.

Mice. 2-photon-targeted electrophysiology and 2-photon calcium imaging was conducted in 2-5 month-old male and female transgenic mice: five Emx1-IRES-Cre;Camk2a-tTA;Ai94 (Emx1-s) mice, one Camk2a-tTA;tetO-GCaMP6s (tetO-s) mouse, three Emx1-IRES-Cre;Camk2atTA;Ai93 (Emx1-f) mice, and four Cux2-CreERT2;Camk2a-tTA;Ai93 (Cux2-f) mice. All four lines drive GCaMP expression primarily in excitatory neurons. In Cux2-CreERT2 mice, Cre and GCaMP expression are enriched in layer 2/3 (Franco et al., 2012; Harris et al., 2014). In Emx1IRES-Cre and Camk2a-tTA mice, GCaMP is expressed throughout cortical layers (Gorski et al., 2002; Wekselblatt et al., 2016). Images showing the pattern of Cre and GCaMP expression in these mouse lines are available via the Transgenic Characterization pages of the Allen Mouse Brain Connectivity Atlas and Allen Brain Observatory: https://connectivity.brainmap.org/transgenic, http://observatory.brain-map.org/visualcoding/transgenic.

Mice of some of the genotypes used here, most notably Emx1-f, can exhibit epileptiform activity (Steinmetz et al., 2017), including overt seizures. Mice with seizures were excluded from the study. However, the spiking patterns of neurons from GCaMP6s and -f lines commonly differed, suggesting that one or more transgenes affected cell or circuit activity (figure 1 - figure supplement 1C). 
Surgery. Mice were anesthetized with either isoflurane $\left(0.75-1.5 \%\right.$ in $\left.\mathrm{O}_{2}\right)$ or urethane $(1.5 \mathrm{~g} / \mathrm{kg}$, $30 \%$ aqueous solution, intraperitoneal injection), then implanted with a metal head-post. A circular craniotomy was performed with skull thinning over the left V1 centering on $1.3 \mathrm{~mm}$ anterior and $2.6 \mathrm{~mm}$ lateral to the Lambda. During surgery, the craniotomy was filled with artificial cerebrospinal fluid (ACSF) containing (in $\mathrm{mM}$ ): $\mathrm{NaCl} 126, \mathrm{KCl} 2.5, \mathrm{NaH}_{2} \mathrm{PO}_{4} 1.25$, $\mathrm{MgCl}_{2}$ 1, $\mathrm{NaHCO}_{3} 26$, glucose $10, \mathrm{CaCl}_{2}$ 2, in $\mathrm{ddH}_{2} \mathrm{O} ; 290 \mathrm{mOsm} ; \mathrm{pH}$ was adjusted to 7.3 with $\mathrm{NaOH}$ to keep the exposed $\mathrm{V} 1$ region from overheating or drying. Durotomy was performed to expose $\mathrm{V} 1$ regions of interest that were free of major blood vessels to facilitate the penetration of recording micropipettes. A thin layer of low melting-point agarose (1-1.3\% in ACSF, SigmaAldrich) was then applied to the craniotomy to control brain motion. The mouse body temperature was maintained at $37^{\circ} \mathrm{C}$ with a feedback-controlled animal heating pad (Harvard Apparatus).

Calcium imaging. Individual GCaMP6+ neurons 100-300 $\mu \mathrm{m}$ below the pial surface of cortex were visualized under adequate anesthesia (Stage III-3) using a Bruker (Prairie) 2-photon microscope and Chameleon Ultra II Ti:sapphire laser (Coherent). Fluorescence excited at 920 $\mathrm{nm}$ wavelength, with $<70 \mathrm{~mW}$ laser power measured after the objective, was collected in two spectral channels using green $(510 / 42 \mathrm{~nm})$ and red $(641 / 75 \mathrm{~nm})$ emission filters (Semrock) to visualize GCaMP6 and the Alexa Fluor 594-containing micropipette, respectively. Fluorescence images of 96-136 x 96-107 pixels and a 19.3-27.3 x 19.3-21.5 $\mu \mathrm{m}$ field of view were acquired at 141.3-158 frames per second through a 16x water-immersion objective lens (Nikon, NA 0.8). Recordings included periods with and without visual stimuli. Mean \pm sem number of pixels per neuron was $1136 \pm 46$.

Electrophysiology. 2-photon targeted cell-attached recording was performed following established protocols (Margrie et al., 2003; Kitamura et al., 2008; Knoblich et al., 2019). Longshank borosilicate (KG-33, King Precision Glass) micropipettes (5-10 M 2 ) were pulled with a P97 puller (Sutter) and filled with ACSF and Alexa Fluor 594 to perform cell-attached recordings on GCaMP6+ neurons. Micropipettes were installed on a MultiClamp 700B headstage (Molecular Devices), which was mounted onto a Patchstar micromanipulator (Scientifica) with an approaching angle of 31 degrees from horizontal plane. Minimal seal resistance was $20 \mathrm{M} \Omega$.

396 Data were acquired under "I = 0" mode (zero current injection) with a Multiclamp 700B, recorded at $40 \mathrm{kHz}$ using Multifunction I/O Devices (National Instruments) and custom software written in 
LabVIEW (National Instruments) and MATLAB (MathWorks). Isoflurane level was intentionally adjusted during recording sessions to keep the anesthesia depth as light as possible, resulting in fluctuation of the firing rates of recorded neurons.

Visual stimulation. Whole-screen sinusoidal static and drifting gratings were presented on a calibrated LCD monitor spanning $60^{\circ}$ in elevation and $130^{\circ}$ in azimuth to the contralateral eye. The mouse's eye was positioned $\sim 22 \mathrm{~cm}$ away from the center of the monitor. For static gratings, the stimulus consisted of 4 orientations ( $45^{\circ}$ increment), 4 spatial frequencies $(0.02$, $0.04,0.08$, and 0.16 cycles per degree), and 4 phases $(0,0.25,0.5,0.75)$ at $80 \%$ contrast in a random sequence with 10 repetitions. Each static grating was presented for 0.25 seconds, with no inter-stimulus interval. A gray screen at mean illuminance was presented randomly a total of 60 times. For drifting gratings, the stimulus consisted of 8 orientations ( $45^{\circ}$ increment), 4 spatial frequency $(0.02,0.04,0.08$, and 0.16 cycle per degree) and 1 temporal frequency $(2 \mathrm{~Hz})$, at $80 \%$ contrast in a random sequence with up to 5 repetitions. Each drifting grating lasted for 2 seconds with an inter-stimulus interval of 2 seconds. A gray screen at mean illuminance was presented randomly for up to 15 times.

Neuron selection. We obtained recordings from 213 neurons and developed a numerical routine to exclude neurons with questionable electrophysiology or fluorescence movies, such as abrupt changes in baseline voltage or AP waveform or image artifacts such as those due to motion, photobleaching or other slow baseline changes. Neurons were accepted for analysis if they passed both electrophysiology and image quality control criteria. Electrophysiology quality control is described in the next section and imaging quality control in the 'image downsampling' section. 145 and 10 neurons were eliminated in the electrophysiology and image quality control steps, leaving 58 neurons. Of these, 10 were excluded from further analysis: red indicator had entered the soma from the pipette in 7 instances; 2 neurons segmented poorly during image analysis; and 1 had a truncated electrophysiology recording. The final data set consisted of 48 neurons.

426

Electrophysiology quality control. Electrophysiology traces were first baseline-subtracted to remove slow drift ( $3^{\text {rd }}$ order Savitzky-Golay filter over 20,001 samples using Matlab sgolayfilt). Action potentials were detected as peaks of amplitude more than ten times the Quiroga threshold (QT), the median(|V(t)|/0.6745). 
432 To develop a numerical routine, a group of human annotators identified 48 'high quality'

433 electrophysiology recordings. We then compiled a large set of descriptive statistics, listed below,

434 and calculated the distribution of each of these statistics in the reference data set, thereby

435 defining an acceptable range expected of high quality recordings. Each descriptive statistic was

436 subsequently computed for recordings from all 213 neurons. Each recording was passed for

437 further analysis if for all metrics it fell within the range spanned by the manually selected data

438 set of 48 recordings.

For each electrophysiology recording, we calculated 35 descriptive statistics.

441 Metrics computed on continuous electrophysiological data:

442 (1) Median relative deviation of the membrane potential (MRDM), the ratio between the median absolute deviation (MAD) and the median: $\mathrm{MRDM}=\mathrm{MAD}(\mathrm{Vm}) / \operatorname{median}(\mathrm{Vm})$

(2) Mean of the Baseline (BL).

(3) Coefficient of variation of the baseline: $\operatorname{std}(B L) /$ mean(BL)

(4) Mean of the baseline noise, approximated by the Quiroga threshold (QT) [15]

(5) Stability of the QT: 1000 10s-intervals were uniformly sampled from each recording, and

(6) $r^{2}$ of linear regression (Matlab regression function) of the 1000 QT samples against the start times of the 10 s segments on which the QT was computed.

452

(7) Slope of linear regression (Matlab regression function) of the 1000 QT samples against the start times of the 10 s segments on which the QT was computed.

455 (9) Slope of linear regression (Matlab's regression function) of the baseline against time.

456 (10) The number of samples for which the baseline-subtracted trace exceeds the Quiroga threshold divided by the number of samples for which it dips below the negative of the

459

460 Metrics computed on the AP time series. Only recordings with > 3 APs were included:

461 (11) Number of APs

462 (12) Maximum likelihood inter-AP interval (Matlab lognfit function).

463 (13) Mean AP amplitude.

464 (14) AP amplitude coefficient of variation.

465 (15) AP amplitude median relative deviation. 
488

489

490

491

492

493

494

495

496

497

498

499

(16) Relative AP amplitude range: (max(amplitude) - $\min ($ amplitude)) / median(amplitude).

(17) AP amplitude max/min ratio: $\max ($ amplitude) / min(amplitude).

(18) Signal-to-noise ratio (SNR), median(amplitude) / QT.

Metrics computed on 2 ms-long AP waveforms, AP time $\pm 1 \mathrm{~ms}$ smoothed with Matlab smooth function with sgolay option:

(19) 'left' width-half-max (LWHM), the mean width at half the amplitude before the detected AP time.

(20) 'right' width-half-max (RWHM), the mean width at half the amplitude after the detected AP time.

(21) full width at half amplitude (FWHM). FWHM $=\mathrm{LWHM}+\mathrm{RWHM}$.

(22) Coefficient of variation of LWHM.

(23) Coefficient of Variation of RWHM.

(24) Coefficient of Variation of FWHM.

(25) $r^{2}$ of linear regression (Matlab regression function) of AP amplitude against AP time.

(26) Slope of linear regression (Matlab regression function) of AP amplitude against AP time.

(27) $r^{2}$ of linear regression (Matlab regression function) of AP FWHM against AP time.

(28) Slope of linear regression (Matlab regression function) of AP FWHM against AP time.

Firing-rate based metrics. Firing rate was estimated by convolution of the AP train with a 1s-long box-car window (Matlab conv function):

(29) Mean firing rate (FR).

(30) Coefficient of variation of FR.

(31) $r^{2}$ of linear regression (Matlab regression function) of firing rate against time.

(32) Slope of linear regression (Matlab regression function) of firing rate against time.

(33) Pearson correlation (Matlab corrcoef function) of BL vs FR.

(34) Pearson correlation (Matlab corrcoef function) between the baseline at AP time points and AP amplitude.

(35) Pearson correlation (Matlab corrcoef function) between the baseline at AP times and the AP FWHM.

Neuropil subtraction, high resolution images. To approximate somatic fluorescence ( $\left.F_{\text {cell_true }}\right)$ without neuropil contamination, a scale version of the neuropil fluorescence $\left(F_{\text {neuropil }}\right)$ was subtracted from each somatic fluorescence trace, after (Akerboom et al., (2012): $F_{\text {cell_true }}(t)=$ 
$500 \quad F_{\text {cell_measured }}(t)-r^{*} F_{\text {neuropil }}(t)$. We determined the optimal scale factor $(r)$ for neurons with

501 GCaMP6f to be 0.82 (see results). We therefore used $r=0.8$ as our default scale factor. For

502 some neurons, $F_{\text {neuropil }}$ was large enough relative to $F_{\text {cell_measured }}$ that $r=0.8$ resulted in negative

503 fluorescence. For these neurons, we set $r$ to $0.7,0.6$, or 0.5. For our data set of 48 GCaMP6s

504 and GCaMP6f neurons, we set $r$ to 0.8 for 40 neurons, to 0.7 for 4 neurons, to 0.6 for 3 neurons,

505 and to 0.5 for 1 neuron.

506

Neuropil subtraction, downsampled images. neuropil subtraction was performed as described for the Allen Brain Observatory (de Vries et al., 2020).

Trace analysis. Electrophysiology and calcium imaging data were analyzed using custom MATLAB and Python scripts. For electrophysiology, Vm was filtered between $250 \mathrm{~Hz}$ and $5 \mathrm{kHz}$,

512 and automated AP detection was performed using a threshold criterion ( $5 \times$ std of $\mathrm{Vm}$ ).

513 For calcium imaging, in-plane motion artifacts were corrected (Dombeck et al., 2007), and

514 neuron/ROI selection was performed using a semi-automatic algorithm (Chen et al., 2013)

515 (kindly provided by Karel Svoboda, Janelia Research Campus). Ring-shaped ROls were used to select GCaMP6-positive excitatory neurons, with GCaMP6 expression typically excluded from

517 the nucleus and restricted to the cytoplasm.

To construct AP-calcium fluorescence response curves, we first identified all isolated AP events. For GCaMP6s, isolated events were separated from previous and subsequent events by $\geq 1000$ $\mathrm{ms}$ and $\geq 500 \mathrm{~ms}$, respectively. For GCaMP6f, isolated events were separated from previous and subsequent events by $\geq 300 \mathrm{~ms}$. One result of finding isolated events is that only a minority of APs were used to construct AP-calcium fluorescence response curves. Within each event, APs were summed over $250 \mathrm{~ms}$. Fluorescence traces were aligned to the first AP in each event, with $\mathrm{t}=0$ preceding the AP by $<1$ frame $(6.3 \mathrm{~ms}$ at $158 \mathrm{~Hz})$. For each event, $\Delta \mathrm{F} / \mathrm{F}=(\mathrm{F}-$

$526 \mathrm{~F}_{0, \text { local }} / \mathrm{F}_{0, \text { global }}$, where $\mathrm{F}_{0 \text {,local }}$ was the mean fluorescence over $100 \mathrm{~ms}$ before the first $\mathrm{AP}$, and

$527 \mathrm{~F}_{0, \text { global }}$ was the minimum $\mathrm{F}_{0 \text {,local }}$ across trials. For GCaMP6s and GCaMP6f, peak $\Delta \mathrm{F} / \mathrm{F}$ was

528 calculated by first finding $t_{\max }$, the time of the maximum $\Delta F / F \leq 500 \mathrm{~ms}$ and $300 \mathrm{~ms}$ after the first 529 AP, respectively. Peak $\Delta F / F$ was the mean $\Delta F / F$ from $t_{\max }-50 \mathrm{~ms}$ to $t_{\max }+50 \mathrm{~ms}$. Bursts of $>5$ 530 APs were excluded from analysis due to the low frequency of such events.

532 Fluorescence-to-photon conversion. Mean and variance of the fluorescence, calculated 533 pixelwise for each image, were linearly related, consistent with shot noise-limited imaging. 
534 The resulting slope and offset of the least squares fit were used to convert fluorescence to

535 number of photons: photons $=(F-(-$ offset/slope $)) /$ slope

536 (http://github.com/Allenlnstitute/QC 2P). To account for different pixel dwell times along the

537 resonant scanning axis, photon gain and offset were computed pixel-by-pixel along the resonant 538 axis.

Trial-to-trial variability. For each neuron, fluorescence was summed over all somatic pixels and converted to photons. For each 1AP event, mean photon count 0.1-0 s before the AP was subtracted. $t_{\max }$, the time of the maximum photon count, was calculated from the mean $1 \mathrm{AP}$ trace. Photon count in each trial was determined at $t_{\max }$. and the $95 \%$ confidence interval was calculated as mean (across trials) \pm 1.96 * $\sqrt{\text { mean peak }}$. The percentage of trials with peak fluorescence outside the $95 \%$ confidence interval was used as a measured of trial-to-trial variability.

Ground truth-optimized event detection. We compared fluorescence traces of the response (1AP or 2AP events) to that of OAP events (Chen et al., 2013). For each recording, the mean response trace was used as the template vector. The template vector was normalized after subtracting the mean to create the unit vector, and the scalar results of projecting the response and noise traces on the unit vector were computed: $r_{i}$ and $n_{i}$ for response and noise scalars, respectively. The detection threshold was defined as the $x^{\text {th }}$ percentile of $n_{i}$ values, where 1-x represented the false positive probability (e.g., $x=95$ for $95^{\text {th }}$ percentile or $5 \%$ false positive probability), and the detection probability (true positive probability) was the fraction of $r_{i}$ values above the detection threshold.

Image downsampling. Fluorescence movies were sub-sampled by a factor of 4 in space, and 5 in time (to pixel size $0.80 \mu \mathrm{m}$ and frame rate $30.3 \mathrm{~Hz}$ ) to match the sampling rate and approximate number of pixels per soma of the Allen Brain Observatory (de Vries et al., 2020). To assess the effect of downsampling on subsequent processing, 20 different approaches were 562 tried in parallel (downsampling starting with the 1st, 2nd, ... 4th pixel x 1st, 2nd, ... 5th frame, 563 respectively). $4 \times 5$ internally identical blocks, one block for each downsampling strategy, were 564 tiled for a total of 400 almost identical regions of interest (ROI) per recording. Segmentation to 565 find somatic regions of interest, demixing of traces from nearby somata, neuropil subtraction 566 and the calculation of $\Delta F / F$ were performed as described for the Allen Brain Observatory (de 567 Vries et al., 2020). 
569 In cases where only the neuron of interest was found during segmentation, trace extraction would yield a family of 400 self-similar traces. To catch cases where segmentation yielded additional objects that were not part of the neuron of interest, additional QC steps were required. The traces were first clustered using DBSCAN (Ester et al., 1996; Schubert et al., 2017), and each cluster median was compared against white noise of the same mean and standard deviation (KS-test) and rejected as artifact if it was not significantly different $(p<0.05)$. In cases where multiple clusters were significantly different from noise, this was either due to multiple neurons being present in the field-of-view, or due to residual motion artifacts resulting in multiple translated copies of the same neuron. To disambiguate these two possibilities, the top three clusters were merged: sums were computed for all six possible combinations (sampled without replacement) of (up to) three most distinct cluster medians, and the combination most significantly correlated with the measured electrophysiological AP train was selected. Correlation significance was determined by building a null distribution of correlations between the cluster medians and 1000 random Poisson trains with a rate matching that of the recorded AP train. If there was no more significant correlation between any cluster median (or sum thereof) and the measured AP train than the 0.5 -th percentile of the null distribution (i.e. $p>$ $0.005)$, the recording was failed. Finally, we eliminated from further analysis $<10$ neurons with an abrupt and sustained (seconds) rise in spike rate and subsequent loss spiking activity out of concern that this activity pattern might indicate a breached plasma membrane.

To compare the noise characteristics of the downsampled images to the Allen Brain Observatory, we computed the robust standard deviation (rSTD), a median-based method with outlier-removal (de Vries et al., 2020). For the Allen Brain Observatory, we analyzed fluorescence over periods in which there were no apparent AP-evoked changes in fluorescence.

\section{ACKNOWLEDGEMENTS}

We are grateful for the Animal Care, Transgenic Colony Management, and Lab Animal Services teams for mouse husbandry, and Carol Thompson and John Phillips for providing project management support. We thank Karel Svoboda, Hod Dana and Tsai-Wen Chen for sharing analysis software. This work was funded by Allen Institute for Brain Science. This work was also supported by grants from National Natural Science Foundation of China (NSFC31871055) and Guangdong Science and Technology Department (2020B1212060018, 2017B030314026 \& 
2018B030334001) to L.L. We thank the Allen Institute founders, Paul G. Allen and Jody Allen, for their vision, encouragement, and support.

\section{COMPETING INTERESTS}

The authors declare no competing financial interests.

\section{DATA AVAILABILITY}

The dataset is available at https://portal.brain-map.org/explore/circuits/oephys.

\section{REFERENCES}

Akerboom, J., Chen, T.-W., Wardill, T.J., Tian, L., Marvin, J.S., Mutlu, S., Calderón, N.C., Esposti, F., Borghuis, B.G., Sun, X.R., et al. (2012). Optimization of a GCaMP calcium indicator for neural activity imaging. J. Neurosci. Off. J. Soc. Neurosci. 32, 13819-13840.

Berens, P., Freeman, J., Deneux, T., Chenkov, N., McColgan, T., Speiser, A., Macke, J.H., Turaga, S.C., Mineault, P., Rupprecht, P., et al. (2018). Community-based benchmarking improves spike rate inference from two-photon calcium imaging data. PLoS Comput. Biol. 14, e1006157.

Chen, T.-W., Wardill, T.J., Sun, Y., Pulver, S.R., Renninger, S.L., Baohan, A., Schreiter, E.R., Kerr, R.A., Orger, M.B., Jayaraman, V., et al. (2013). Ultrasensitive fluorescent proteins for imaging neuronal activity. Nature 499, 295-300.

Daigle, T.L., Madisen, L., Hage, T.A., Valley, M.T., Knoblich, U., Larsen, R.S., Takeno, M.M., Huang, L., Gu, H., Larsen, R., et al. (2018). A Suite of Transgenic Driver and Reporter Mouse Lines with Enhanced Brain-Cell-Type Targeting and Functionality. Cell 174, 465-480.e22.

Dana, H., Chen, T.-W., Hu, A., Shields, B.C., Guo, C., Looger, L.L., Kim, D.S., and Svoboda, K. (2014). Thy1-GCaMP6 transgenic mice for neuronal population imaging in vivo. PloS One 9 , e108697.

Dana, H., Mohar, B., Sun, Y., Narayan, S., Gordus, A., Hasseman, J.P., Tsegaye, G., Holt, G.T., Hu, A., Walpita, D., et al. (2016). Sensitive red protein calcium indicators for imaging neural activity. ELife 5.

Dana, H., Sun, Y., Mohar, B., Hulse, B.K., Kerlin, A.M., Hasseman, J.P., Tsegaye, G., Tsang, A., Wong, A., Patel, R., et al. (2019). High-performance calcium sensors for imaging activity in neuronal populations and microcompartments. Nat. Methods 16, 649-657.

Deneux, T., Kaszas, A., Szalay, G., Katona, G., Lakner, T., Grinvald, A., Rózsa, B., and Vanzetta, I. (2016). Accurate spike estimation from noisy calcium signals for ultrafast threedimensional imaging of large neuronal populations in vivo. Nat. Commun. 7, 12190.

Dombeck, D.A., Khabbaz, A.N., Collman, F., Adelman, T.L., and Tank, D.W. (2007). Imaging large-scale neural activity with cellular resolution in awake, mobile mice. Neuron 56, 43-57. 
639

640

641

642

643

644

645

646

647

648

649

650

651

652

653

654

655

656

657

658

659

660

661

662

663

664

665

666

667

668

669

670

671

672

673

674

675

676
Ester, M., Kriegel, H.-P., Sander, J., and Xu, X. (1996). A Density-Based Algorithm for Discovering Clusters in Large Spatial Databases with Noise. In Proceedings of the Second International Conference on Knowledge Discovery and Data Mining, (AAAI Press), pp. 226-231.

Franco S.J., Gil-Sanz C., Martinez-Garay I., Espinosa A., Harkins-Perry S.R., Ramos C., Muller U. (2012). Fate-restricted neural progenitors in the mammalian cerebral cortex. Science 337, 746-749.

Friedrich, J., Zhou, P., and Paninski, L. (2017). Fast online deconvolution of calcium imaging data. PLOS Comput. Biol. 13, e1005423.

Gorski J.A., Talley T., Quu M., Puelles L., Rubenstein J.L.R., Jones K.R. (2002). Cortical excitatory neurons and glia, but not GABAergic neurons, are produced in the Emx1-expressing lineage. J Neurosci 22, 6309-6314.

Harris J.A., Hirokawa K.E., Sorensen S.A., Gu H., Mills M., Ng L.L., Bohn P., Mortrud M., Ouellette B., Kidney J., Smith K.A., Dang C., Sunkin S., Bernard A., Oh S.W., Madisen L., Zeng $\mathrm{H}$. (2014). Anatomical characterization of Cre driver mice for neural circuit mapping and manipulation. Front Neural Circuits 8.

Jewell, S.W., Hocking, T.D., Fearnhead, P., and Witten, D.M. (2020). Fast nonconvex deconvolution of calcium imaging data. Biostatistics 21, 709-726.

Jun, J.J., Steinmetz, N.A., Siegle, J.H., Denman, D.J., Bauza, M., Barbarits, B., Lee, A.K., Anastassiou, C.A., Andrei, A., Aydın, Ç., et al. (2017). Fully integrated silicon probes for highdensity recording of neural activity. Nature 551, 232-236.

Kazemipour, A., Novak, O., Flickinger, D., Marvin, J.S., Abdelfattah, A.S., King, J., Borden, P.M., Kim, J.J., Al-Abdullatif, S.H., Deal, P.E., et al. (2019). Kilohertz frame-rate two-photon tomography. Nat. Methods 16, 778-786.

Kerlin, A.M., Andermann, M.L., Berezovskii, V.K., and Reid, R.C. (2010). Broadly tuned response properties of diverse inhibitory neuron subtypes in mouse visual cortex. Neuron 67 , 858-871.

Kitamura, K., Judkewitz, B., Kano, M., Denk, W., and Häusser, M. (2008). Targeted patch-clamp recordings and single-cell electroporation of unlabeled neurons in vivo. Nat. Methods 5, 61-67.

Knoblich, U., Huang, L., Zeng, H., and Li, L. (2019). Neuronal cell-subtype specificity of neural synchronization in mouse primary visual cortex. Nat. Commun. 10, 2533.

Luo, L., Callaway, E.M., and Svoboda, K. (2018). Genetic Dissection of Neural Circuits: A Decade of Progress. Neuron 98, 256-281.

Madisen, L., Garner, A.R., Shimaoka, D., Chuong, A.S., Klapoetke, N.C., Li, L., van der Bourg, A., Niino, Y., Egolf, L., Monetti, C., et al. (2015). Transgenic mice for intersectional targeting of neural sensors and effectors with high specificity and performance. Neuron 85, 942-958.

Margrie, T.W., Meyer, A.H., Caputi, A., Monyer, H., Hasan, M.T., Schaefer, A.T., Denk, W., and Brecht, M. (2003). Targeted whole-cell recordings in the mammalian brain in vivo. Neuron 39, 911-918. 
677 Pachitariu, M., Stringer, C., and Harris, K.D. (2018). Robustness of Spike Deconvolution for 678 Neuronal Calcium Imaging. J. Neurosci. 38, 7976-7985.

679 Schubert, E., Sander, J., Ester, M., Kriegel, H.P., and Xu, X. (2017). DBSCAN Revisited, 680 Revisited: Why and How You Should (Still) Use DBSCAN. ACM Trans Database Syst 42.

681 Steinmetz, N.A., Buetfering, C., Lecoq, J., Lee, C.R., Peters, A.J., Jacobs, E.A.K., Coen, P., 682 Ollerenshaw, D.R., Valley, M.T., de Vries, S.E.J., et al. (2017). Aberrant Cortical Activity in 683 Multiple GCaMP6-Expressing Transgenic Mouse Lines. ENeuro 4.

684 Theis, L., Berens, P., Froudarakis, E., Reimer, J., Román Rosón, M., Baden, T., Euler, T., 685 Tolias, A.S., and Bethge, M. (2016). Benchmarking Spike Rate Inference in Population Calcium 686 Imaging. Neuron 90, 471-482.

687 Villette, V., Chavarha, M., Dimov, I.K., Bradley, J., Pradhan, L., Mathieu, B., Evans, S.W., 688 Chamberland, S., Shi, D., Yang, R., et al. (2019). Ultrafast Two-Photon Imaging of a High-Gain 689 Voltage Indicator in Awake Behaving Mice. Cell 179, 1590-1608.e23.

690 de Vries, S.E.J., Lecoq, J.A., Buice, M.A., Groblewski, P.A., Ocker, G.K., Oliver, M., Feng, D., 691 Cain, N., Ledochowitsch, P., Millman, D., et al. (2020). A large-scale standardized physiological 692 survey reveals functional organization of the mouse visual cortex. Nat. Neurosci. 23, 138-151.

693 Wei, Z., Lin, B.-J., Chen, T.-W., Daie, K., Svoboda, K., and Druckmann, S. (2019). A

694 comparison of neuronal population dynamics measured with calcium imaging and 695 electrophysiology (Neuroscience).

696 Wekselblatt, J.B., Flister, E.D., Piscopo, D.M., and Niell, C.M. (2016). Large-scale imaging of 697 cortical dynamics during sensory perception and behavior. J. Neurophysiol. 115, 2852-2866.

698 Wu, J., Liang, Y., Chen, S., Hsu, C.-L., Chavarha, M., Evans, S.W., Shi, D., Lin, M.Z., Tsia, 699 K.K., and Ji, N. (2020). Kilohertz two-photon fluorescence microscopy imaging of neural activity 700 in vivo. Nat. Methods 17, 287-290. 
701 Table 1. Dataset. Sample size for each mouse line (see also Figure 1 - figure supplement 1).

\begin{tabular}{|c|c|c|c|c|c|c|}
\hline Mouse line & Acronym & GECI & Mice & Cells & $\begin{array}{c}\text { Recording } \\
\text { duration }\end{array}$ & $\begin{array}{c}\text { APs per } \\
\text { neuron }\end{array}$ \\
\hline $\begin{array}{c}\text { Emx1-IRES-Cre; } \\
\text { Camk2a-tTA;Ai94 }\end{array}$ & Emx1-s & GCaMP6s & 5 & 21 & $241 \pm 32 \mathrm{~s}$ & $478 \pm 121$ \\
\hline $\begin{array}{c}\text { Camk2a-tTA; } \\
\text { tetO-GCaMP6s }\end{array}$ & tetO-s & GCaMP6s & 1 & 4 & $347 \pm 108 \mathrm{~s}$ & $348 \pm 71$ \\
\hline $\begin{array}{c}\text { Cux2-CreERT2; } \\
\text { Camk2a-tTA;Ai93 }\end{array}$ & Cux2-f & GCaMP6f & 3 & 12 & $300 \pm 79 \mathrm{~s}$ & $484 \pm 112$ \\
\hline $\begin{array}{c}\text { Emx1-IRES-Cre; } \\
\text { Camk2a-tTA;Ai93 }\end{array}$ & Emx1-f & GCaMP6f & 4 & 11 & $201 \pm 23 \mathrm{~s}$ & $219 \pm 38$ \\
\hline
\end{tabular}

702

703 
(A) Experimental design. (B) Fluorescence and Vm traces from an exemplar Emx1-s neuron.

(C) 5 seconds of data from the neuron in panel B, showing a 2AP, a 1 AP and a 3 AP event. Pre- and post-AP exclusion windows, used to separate events, are illustrated for each event.

Figure 1 - figure supplement 1. Mouse age, cell depth, and firing rate. (A) Mouse age. (B) Cell depths. (C) For each neuron, percentage of APs contained within 1-5 AP events. Figure 2. Neuropil subtraction optimized for 1AP detection. (A) Effect of changing neuropil subtraction on detection for exemplar GCaMP6s and GCaMP6f neurons. Upper plots: family of ROC curves. Each curve illustrates detection probability for true APs against probability of false positives as detection threshold is changed, for 1 AP events. False positives were calculated from time windows with no APs. Each ROC curve represents a different value of $r$. Lower plots: area under the ROC curve as a function of $r$. Grey symbols represent value of $r$ for which $r{ }^{*} F_{\text {neuropil }}(t)$ was greater than $F_{\text {cell_measured }}(t)$, resulting in a negative F0 and inversion of the $\Delta F / F$ trace. (B) Distribution of $r$ values for 20 GCaMP6f neurons.

Figure 2 - figure supplement 1. Simulated effect of neuropil subtraction on event detection. (A) Simulated cell and neuropil traces. The neuropil trace contained transients that were (1) associated with cell transients and (2) between cell transients, and amplitudes were scaled by the neuropil contamination ratio $r(r=0.3)$ relative to the cell amplitudes. (B) (Left) ROC curves for classifying cell amplitudes, where $r$ was varied from 0 to 1 for neuropil correction. The detection threshold was defined as the $x^{\text {th }}$ percentile of noise amplitudes (amplitudes of the neuropil trace that were between cell transients), where 1-x represented the false positive probability, and the detection probability (true positive probability) was the fraction of estimated cell amplitudes (amplitudes of the summed trace that were associated with cell transients) above the detection threshold. (Right) Area under ROC curves as a function of $r$.

\section{Figure 3. AP-evoked calcium transients in 4 mouse lines.} events for an exemplar Emx1-s neuron (42 1AP events, 38 2AP events, 163 AP events) and an exemplar Emx1-f neuron (23 1AP events, 12 2AP events, 11 3AP events). (B) Mean 
fluorescence traces and fits (sum of two exponentials) for 1-5 AP events for the two neurons in A. (C) Mean \pm sem peak DF/F, rise time constant, and decay time constant for four mouse lines. Number of neurons for 1-5 AP events were: 15, 16, 14, 9, 6 for Emx1-s; 4, 4, 4, 0, 0 for tetO-s; 10, 10, 10, 5, 4 for Emx1-f; 9, 9, 7, 4, 2 for Cux2-f. Asterisks indicate differences between mouse lines $(p<0.05$, one-way ANOVA).

\section{Figure 3 - figure supplement 1 . Trial-to-trial variability of 1 AP events}

Fraction of trials in which the peak of the fluorescence transient exceeded the range expected from Poisson noise, a measure of trial-to-trial variability. (A) Example 1AP event for an Emx1-s neuron, with fluorescence in units of photons. Dashed line: mean, across trials, of peak fluorescence. Grey: 95\% confidence interval for Poisson-distributed noise. (B) Amplitudes of $1 \mathrm{AP}$ events for 30 trials. Dashed line and grey area: mean and 95\% confidence interval. (C) Mean \pm SEM percentage of trials with peak $\Delta \mathrm{F}$ outside the $95 \%$ confidence interval, for each mouse line. In all four mouse lines, peak fluorescence exceeded the 95\% confidence interval for Poisson-distributed noise in $>>5 \%$ of $1 \mathrm{AP}$ trials. The fraction of trials outside the confidence interval correlated with resting fluorescence, likely the result of different illumination intensities across experiments.

\section{Figure 4. Downsampling mimics large field-of-view images}

(A) Original, high spatial and temporal resolution GCaMP6f image of an exemplar Cux2-f neuron. (B) 1, 2 and 3AP traces for the same neuron. Thin lines, individual trials; thick line, mean. Dashed line, $\Delta F / F=0$. (C) Downsampled image of the same neuron. (D) Traces from the downsampled neuron. (E) Normalized distribution of baseline noise (rSTD) for 48 downsampled neurons (red) and 11,816 layer 2/3 neurons from Emx1-f and Cux2-f mice in the Allen Brain Observatory (blue).

\section{Figure 4 - figure supplement 1. Example fluorescence traces from image quality control} procedures, implemented during image downsampling.

\section{(A) Median of traces extracted from an example segmentation in which only the neuron of} interest was found in each image during segmentation. The trace is significantly different from noise (KS test, $\mathrm{p}=1.8 \times 10^{-40}$ ). (B) Median of traces extracted from an example segmentation in which only the neuron of interest was found in each image during segmentation. The trace is not significantly different from noise (KS test, $p=0.072$ ). (C) Medians of traces extracted from an example segmentation in which three regions of interest were identified. Each trace is 
significantly different from noise (KS tests, $p=7.7 \times 10^{-152}, 1.6 \times 10^{-19}, 2.2 \times 10^{-25}$ ). The sum of these three cluster medians had the highest correlation with the firing rate and was therefore used as the $\Delta F / F$ trace for this recording in the dataset. Scale bards apply to A-C. (D) Example fluorescence trace with an abrupt and sustained rise in fluorescence and in spike rate and subsequent loss of spiking activity. This neuron was manually removed from the dataset.

\section{Figure 5. Downsampling affects event detection.}

(A-C) Event detection from images at high spatial and temporal resolution. (A) ROC curves for 1 AP events in 42 neurons, organized by mouse line. Neuropil subtraction was performed with $r=$ 0.8 where possible (see Methods). Numbers of neurons were $18 \mathrm{Emx1-s,} 3$ tetO-s, $11 \mathrm{Cux2-f,} 9$ Emx1-f. (B) Mean ROC curves for the four mouse lines. (C) Event detection probabilities for 1 and 2 AP events. Bars represent mean \pm sem. (D-F) Equivalent plots for the same neurons after downsampling.

\section{Figure 6. Performance of spike inference algorithms on high resolution and} downsampled images. (A) Results from an exemplar Cux2-f neuron, at high resolution. Fluorescence and AP rate from electrophysiology (black). Below, APs per image frame estimated with 3 spike inference algorithms: MLspike (blue), Exact L0 (purple), and nonnegative deconvolution (NND, orange). (B) Pearson correlation coefficient ( $r$ ) and Matthews correlation coefficient (MCC) for the three algorithms for each mouse line. $300 \mathrm{~ms}$ bins. (C) ROC curves, reporting probabilities of detecting true and false events in each time bin. Thin lines: individual neurons. Thick lines: mean across neurons. $300 \mathrm{~ms}$ bins. (D-F) Equivalent plots for downsampled images.

\section{Figure 6 - figure supplement 1. Effect of bin duration on measures of spike inference} algorithm performance. (A) ROC curves for MLspike (blue), exact LO (purple), and NND (orange) algorithms after binning of spike probabilities into 33, 100, 200, 300 and 500 ms bins, for downsampled images resampled to $150 \mathrm{~Hz}$. (B and C) Pearson correlation coefficients ( $r$ ) and Matthews correlation coefficients (MCC) for each bin duration.

\section{Figure 7. Performance of blind spike inference algorithms for 1-5 AP events.}

$$
\text { (A) Mean } \pm \text { SEM detection probabilities at } 1 \% \text { false positive probability for high resolution }
$$
images. (B) Mean \pm SEM detection probabilities at $1 \%$ false positive probability for downsampled images. (C) Frequency of 0-5 AP events for $250 \mathrm{~ms}$ bins for each mouse line. 
807 Figure 7 - figure supplement 1. Upsampling enhances performance of unconstrained

808 non-negative deconvolution. Performance of the NND algorithm when applied to fluorescence

809 traces from downsampled images (frame rate $30 \mathrm{~Hz}$ ) and to the same fluorescence traces after

810 upsampling to $150 \mathrm{~Hz} .100 \mathrm{~ms}$ bins. (A) Pearson r and Matthews correlation coefficient (MCC).

811 (B) ROC curves.

812

813 Figure 7 - figure supplement 2. Autocalibration enhanced performance of MLspike.

814 Performance of MLspike with and without use of the autocalibration procedure, applied to

815 downsampled images, with fluorescence traces upsampled to a frame rate of $158 \mathrm{~Hz} .100 \mathrm{~ms}$

816 bins. (A) Pearson $r$ and Matthews correlation coefficient (MCC). (B) ROC curves.

817

818 Figure 7 - figure supplement 3. Comparison of spike inference with blind and ground

819 truth AP-optimized algorithms. (A) Mean \pm SEM 1AP event detection probability at $1 \%$ false

820 positive probability for ground-truth optimized and blind algorithms (used in figure 5 and in

821 figures 6 and 7, respectively). Traces were selected as for Figure 5. (B) 1AP events detected by

822 each blind algorithm as a fraction of those detected by the ground truth-optimized algorithm, at

$8231 \%$ false positive probability. 
figure 1
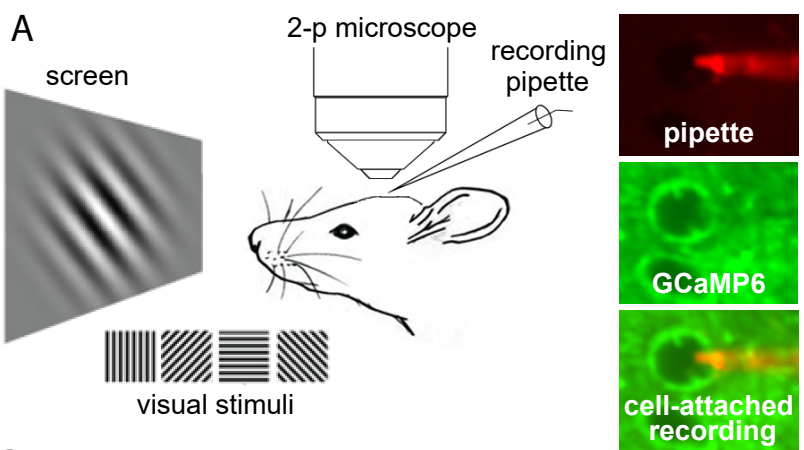

B calcium imaging
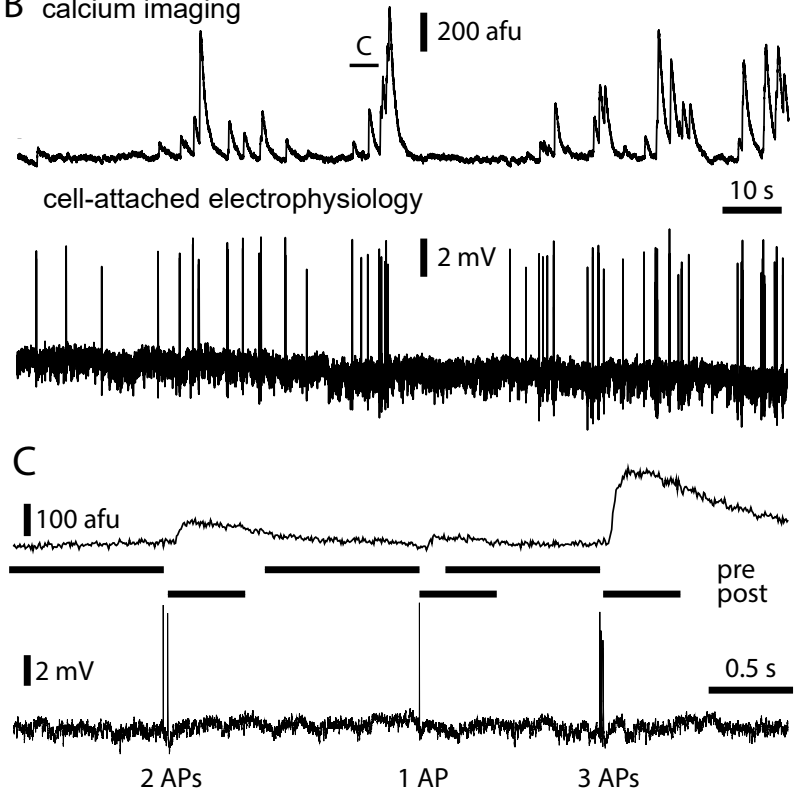


\section{figure 1 supplement 1}

A

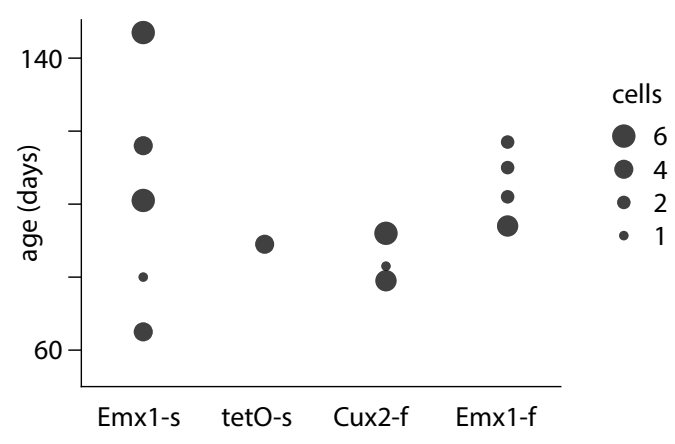

B

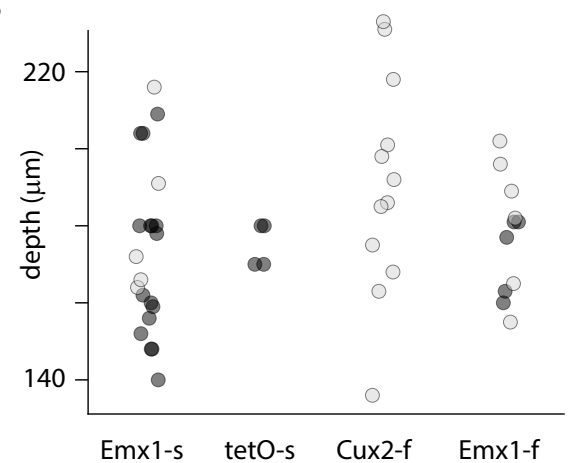

C

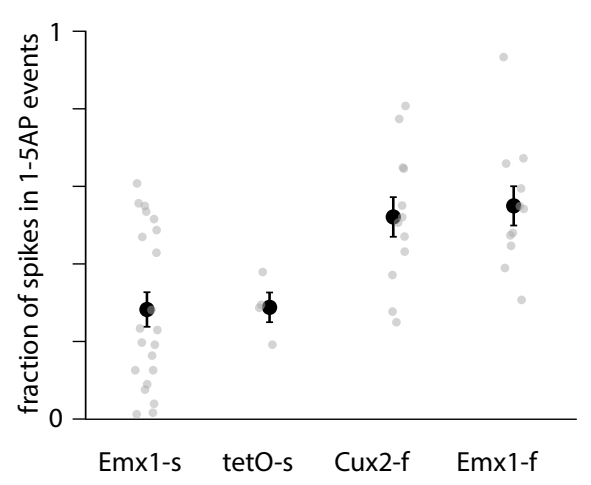

spontaneous

visual stimuli 
figure 2
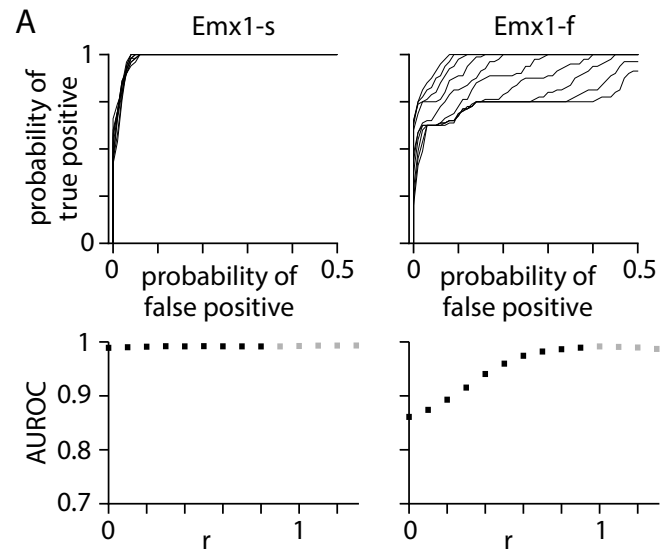

B

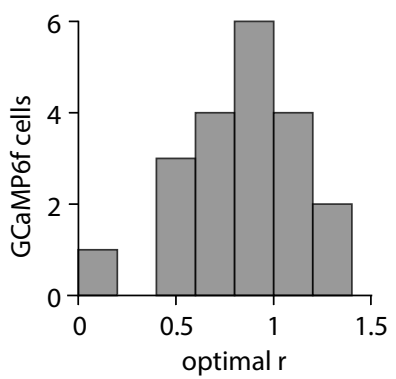


figure 2, figure supplement 1

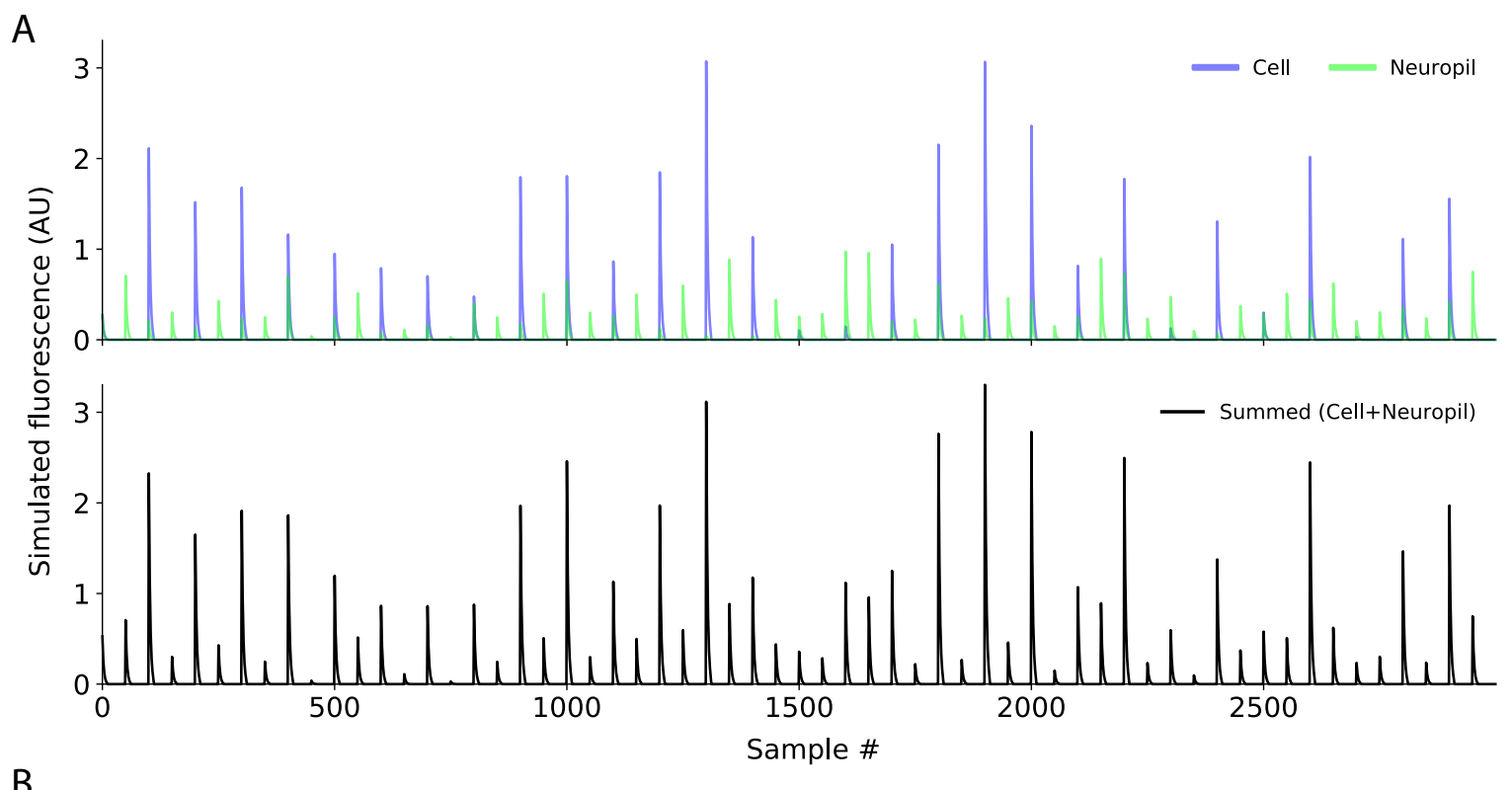

B
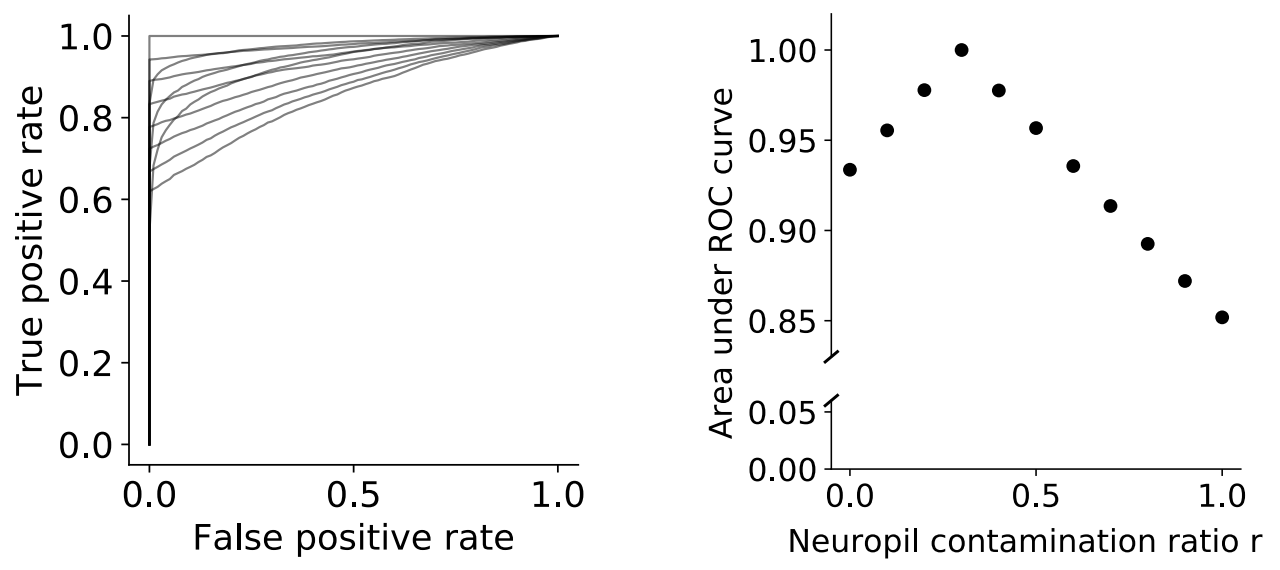
figure 3

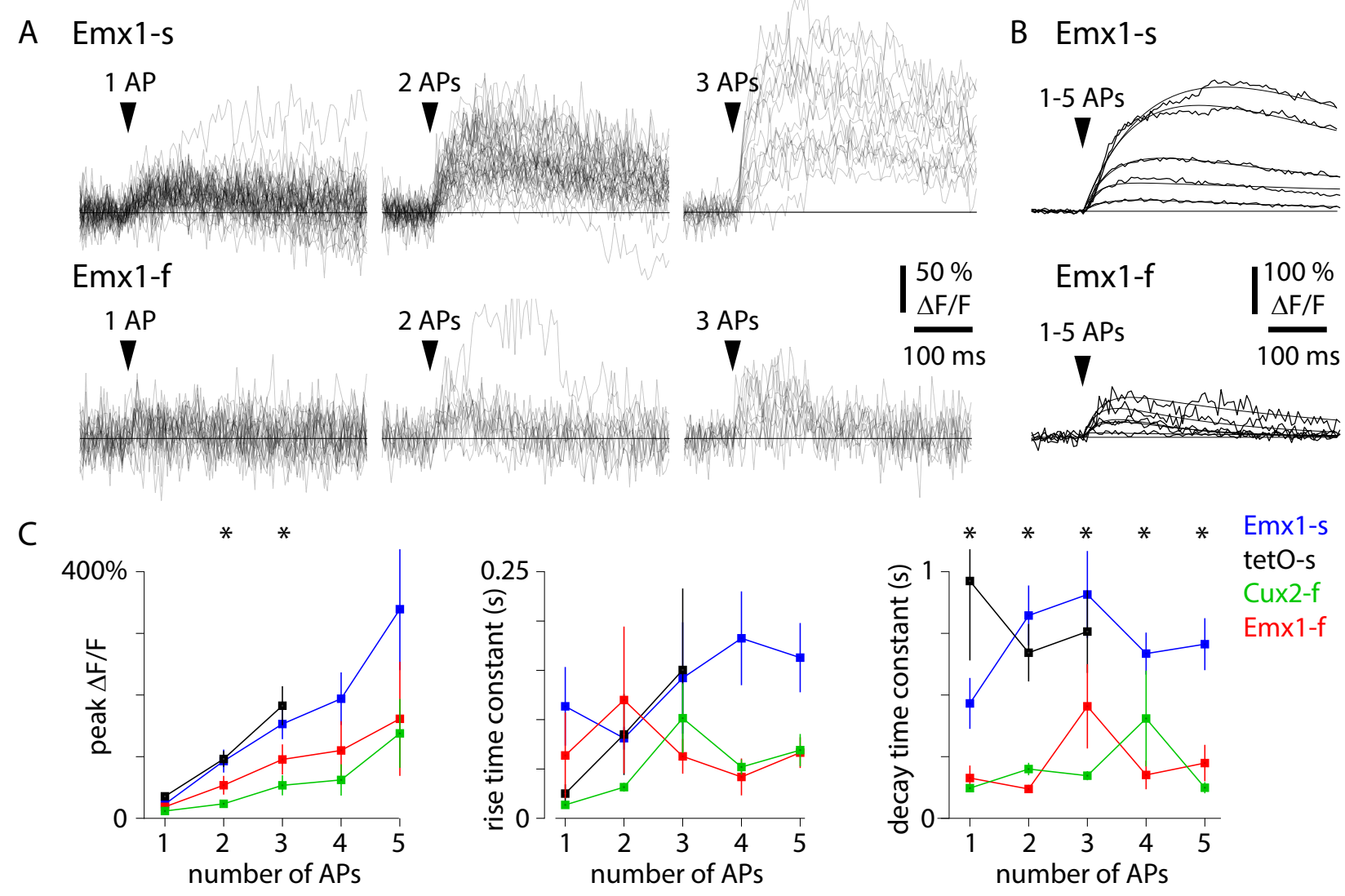


figure 3 , figure supplement 1
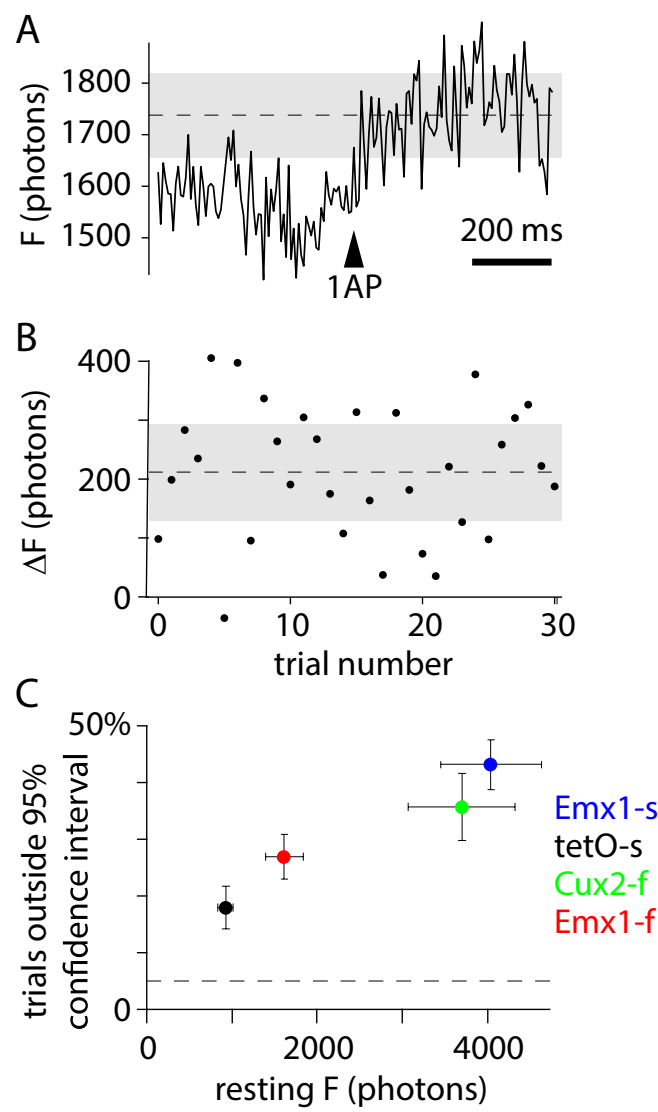


\section{figure 4}
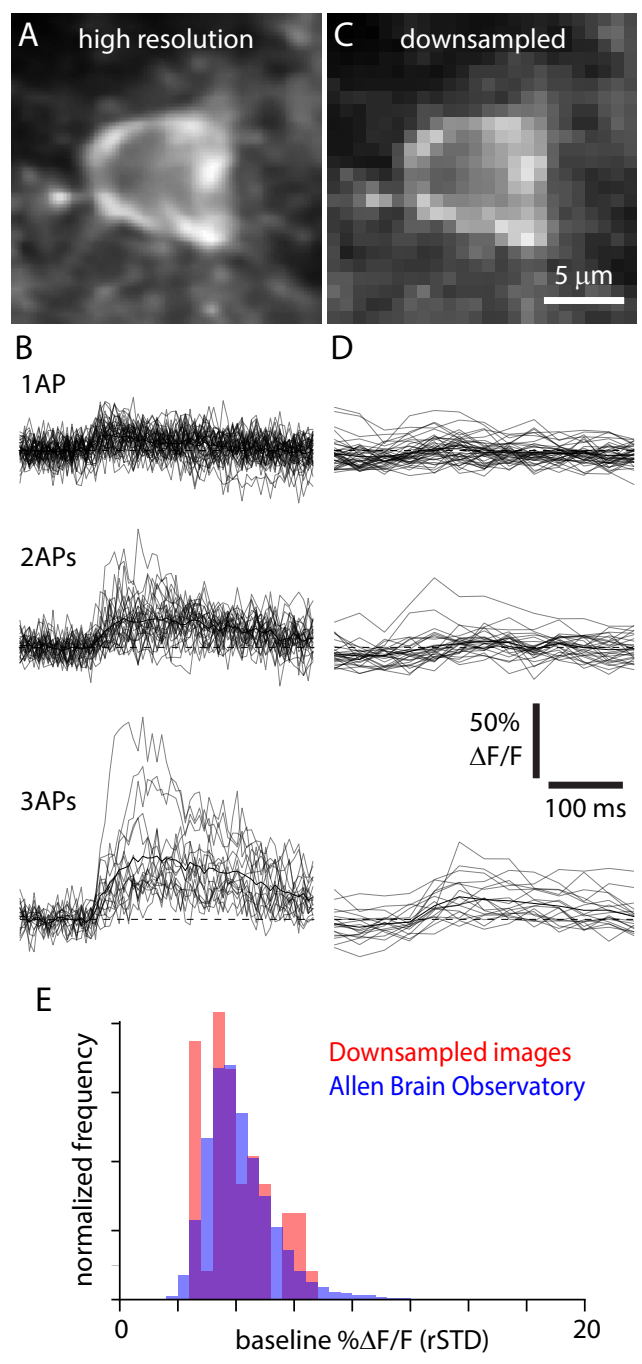
figure 4 - figure supplement 1

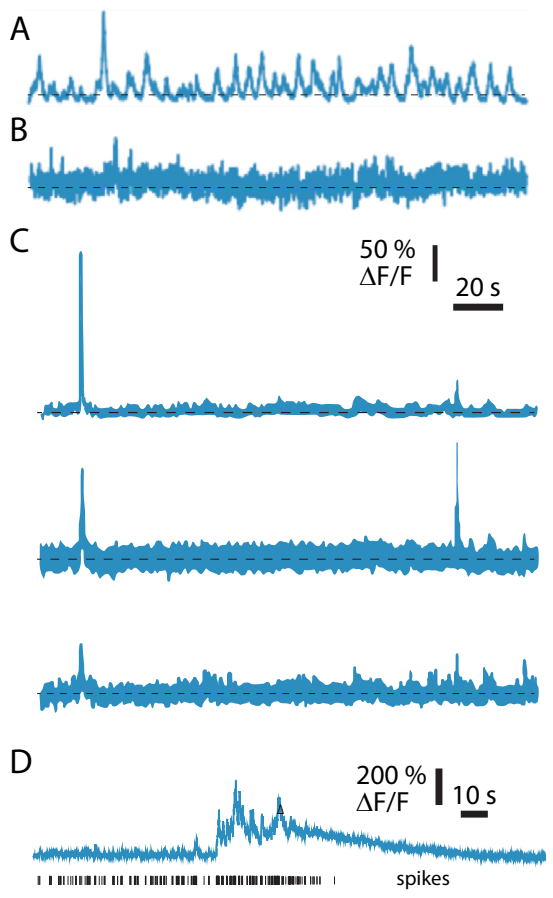




\section{figure 5}
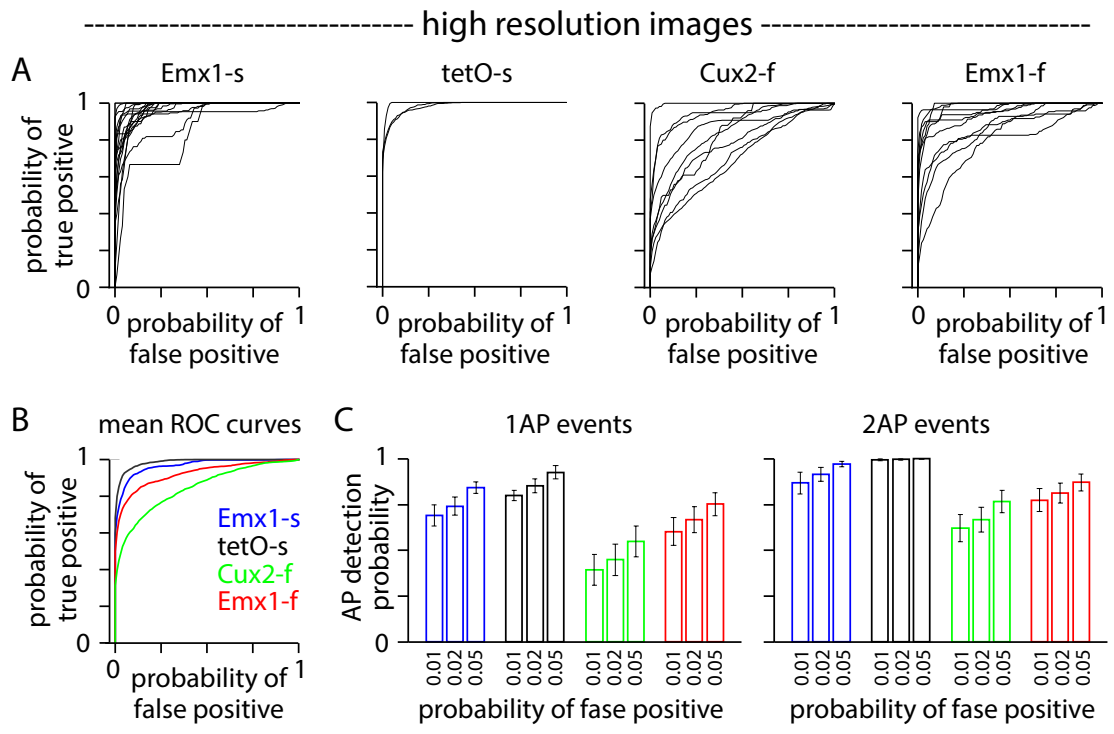

2AP events

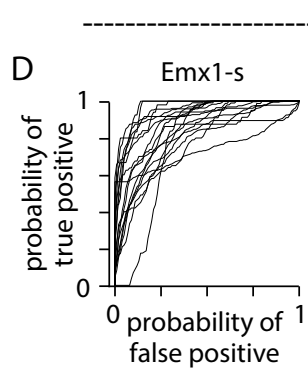

downsampled images
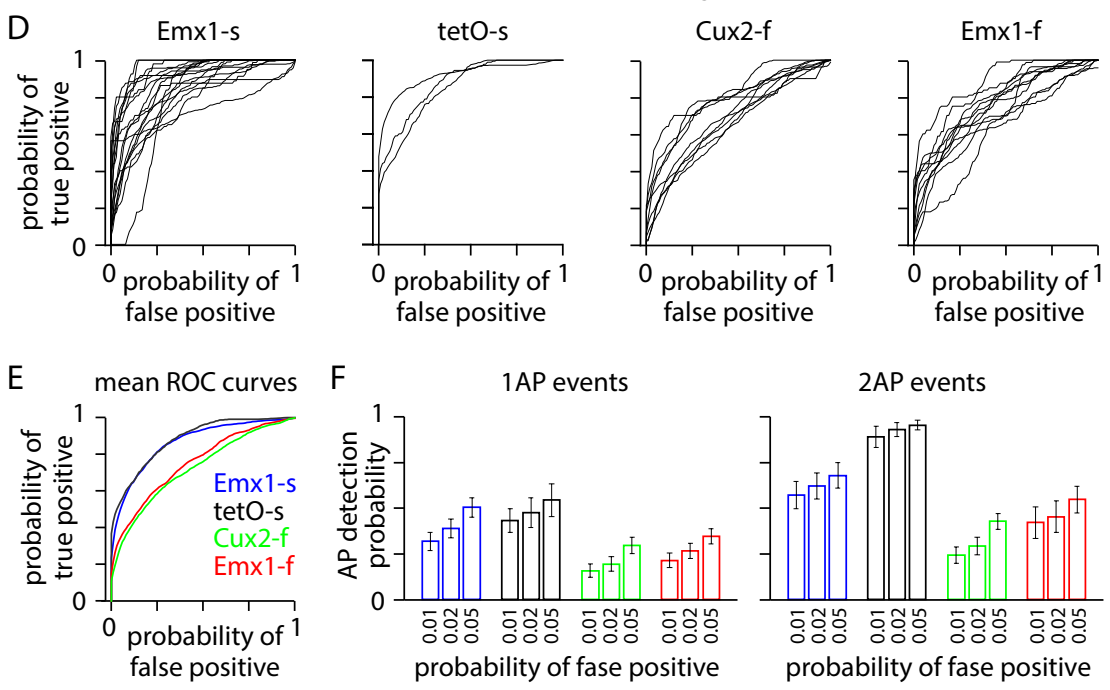
figure 6

A high resolution images fluorescence AP rate

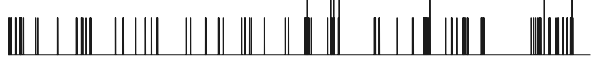

inferred AP rate, NND

inferred AP rate, Exact LO

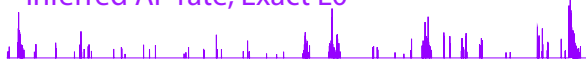

inferred AP rate, MLspike

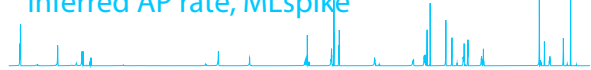

B
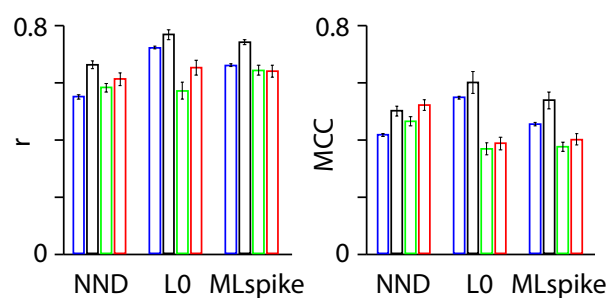

C
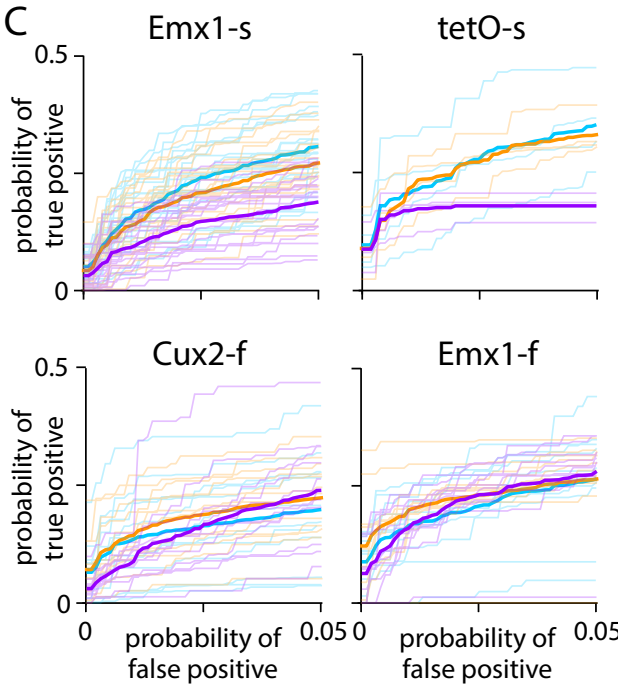

downsampled images
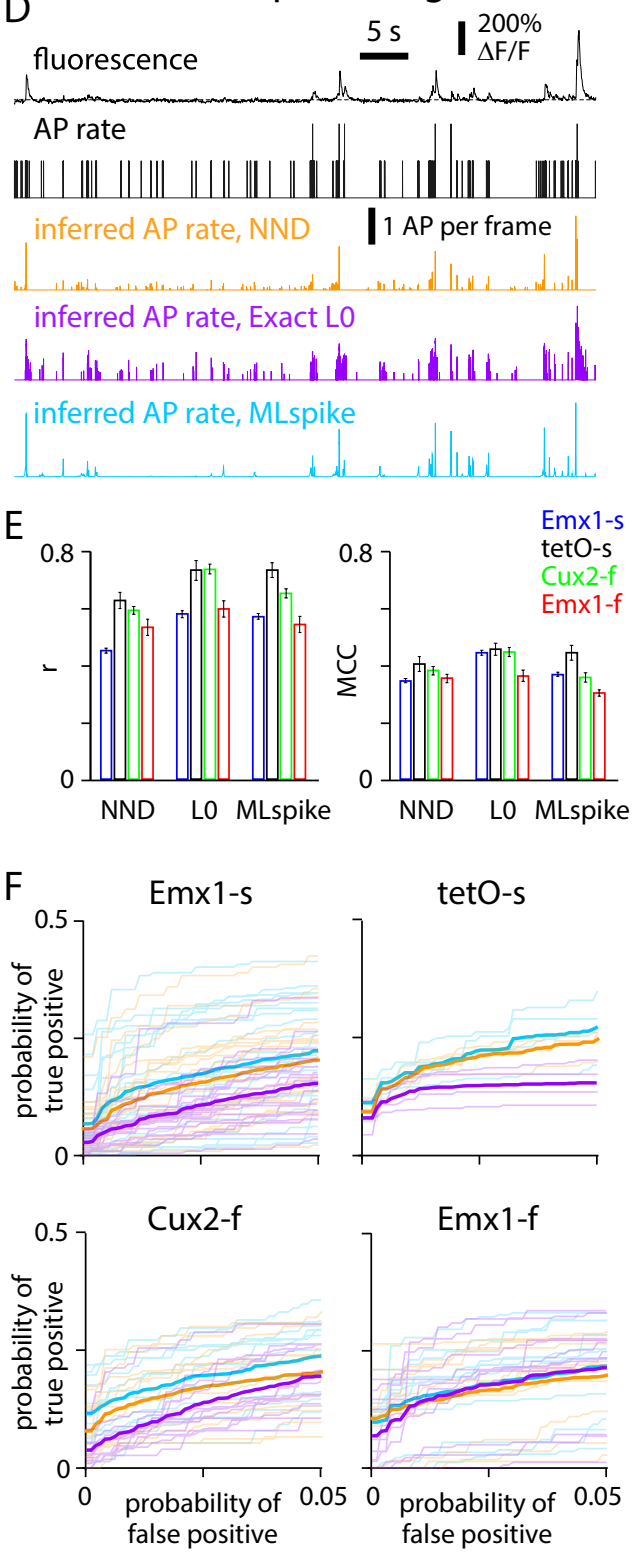

Emx1-f

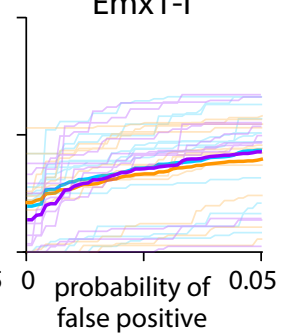


figure 6 supplement 1




figure 7

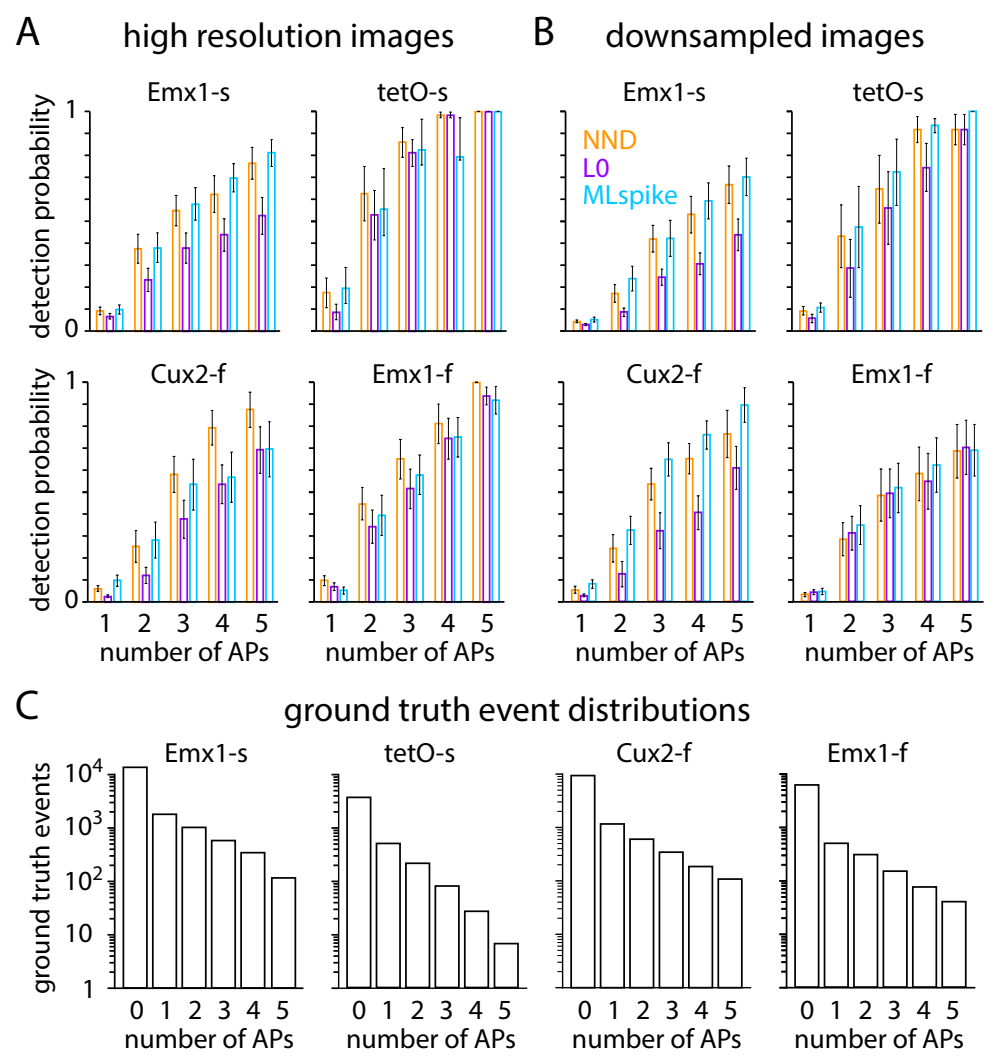




\section{figure 7, figure supplement 1}

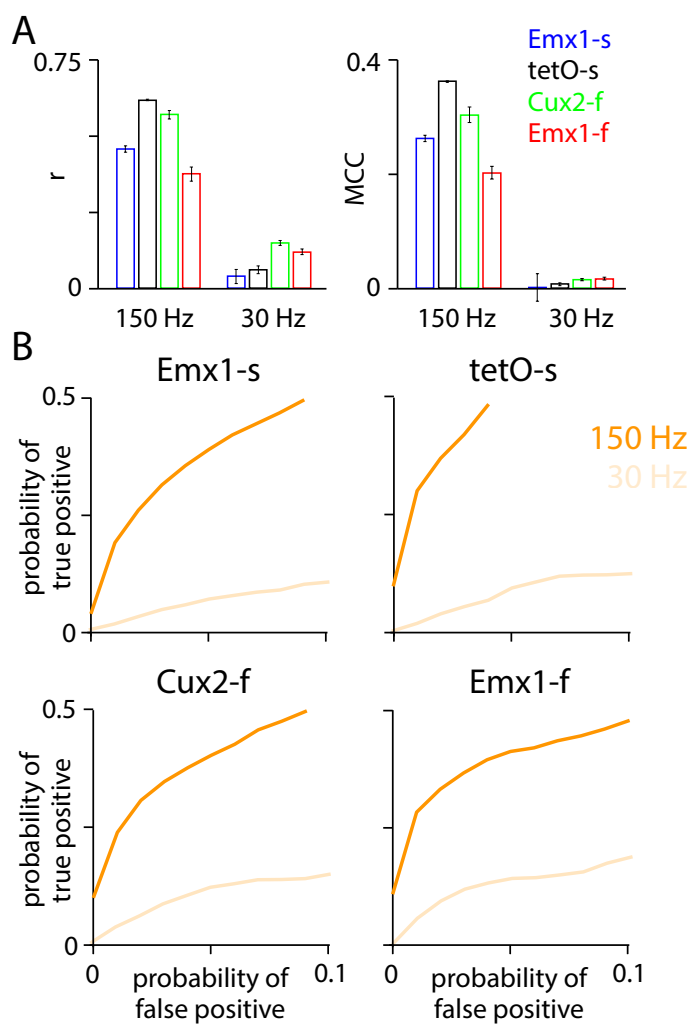


figure 7, figure supplement 2
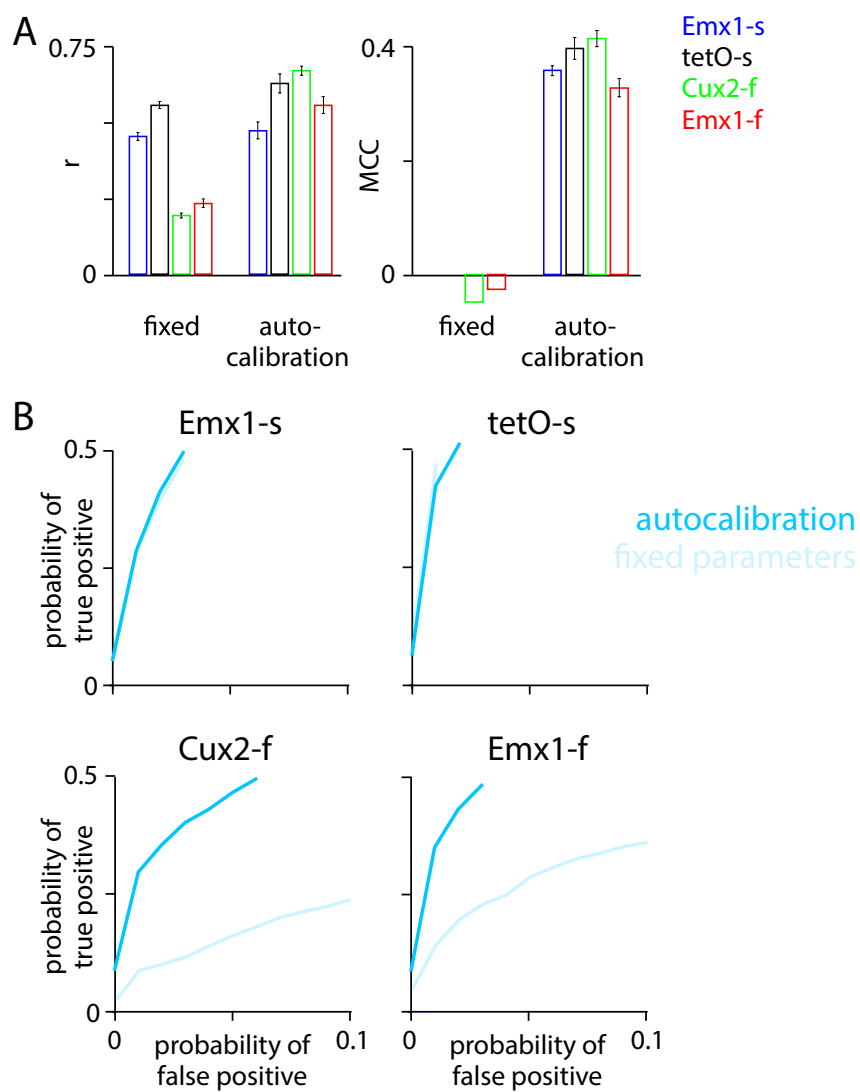


\section{figure 7 - figure supplement 3}

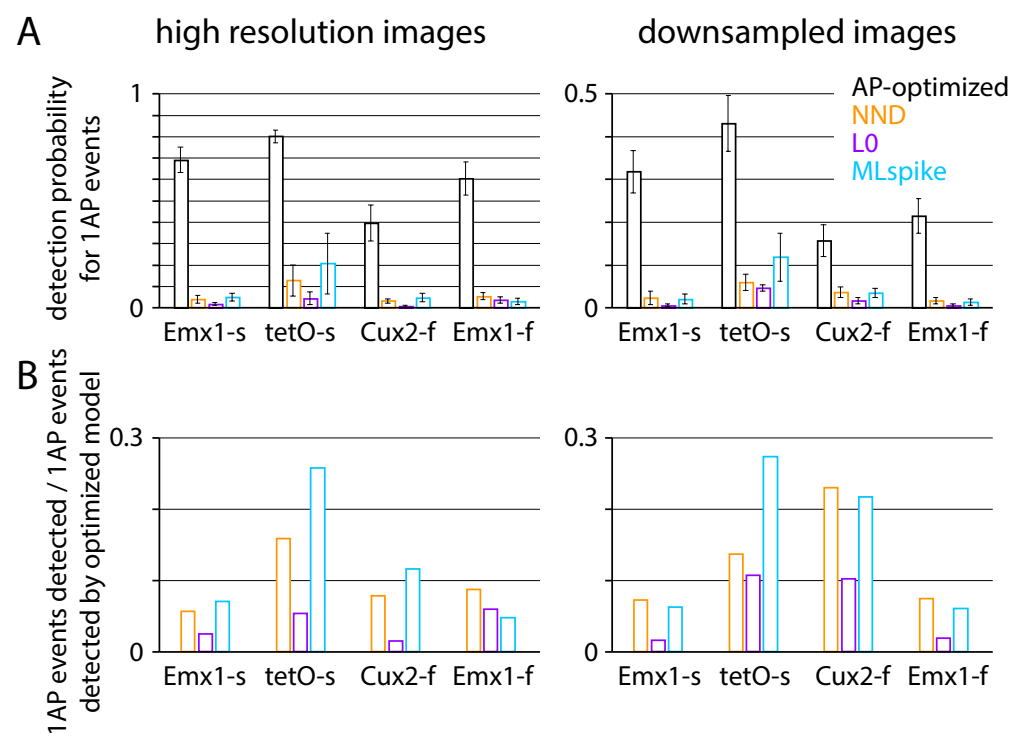

